\title{
1. Politique du secrétariat d'Etat a l'économie en matière de promotion des investissements
}

Gérard Perroulaz, Andreas Ragaz, Hans-Peter Egler, Monika Egger, Susanne Grossmann, Peter Bosshard et Nadine Keim

\section{OpenEdition}

\section{Journals}

Édition électronique

URL : http://journals.openedition.org/aspd/869

DOI : 10.4000/aspd.869

ISSN : 1663-9669

Éditeur

Institut de hautes études internationales et du développement

Édition imprimée

Date de publication : 1 avril 2001

Pagination : 5-51

ISSN : $1660-5934$

Référence électronique

Gérard Perroulaz, Andreas Ragaz, Hans-Peter Egler, Monika Egger, Susanne Grossmann, Peter Bosshard et Nadine Keim, «1. Politique du secrétariat d'Etat a l'économie en matière de promotion des investissements ", Annuaire suisse de politique de développement [En ligne], 20 | 2001, mis en ligne le 27 août 2012, consulté le 07 septembre 2020. URL : http://journals.openedition.org/aspd/869 DOI : https://doi.org/10.4000/aspd.869 


\section{POLTIQUE DU SECRÉTARIAT D'ÉTAT À L'ÉCONOMIE EN MATIËRE DE PROMOTION DES INVESTISSEMENTS}

\subsection{PRÉSENTATION DES NOUVEAUX INSTRUMENTS DE PROMOTION DES INVESTISSEMENTS}

\section{Gérard Perroulaz}

La Loi sur la coopération au développement du 19 mars 1976 stipulait déjà (à l'article 6) que la coopération au développement peut revêtir la forme de «mesures en vue d'encourager l'engagement de ressources du secteur privé, telles que les investissements ». Plusieurs documents donnant les orientations générales de l'aide suisse ont depuis réaffirmé et précisé les mesures de coopération susceptibles de soutenir les efforts du secteur privé1.

La base légale sur la coopération avec les pays de l'Europe de l'Est cite aussi comme objectif la nécessité d'une coopération en vue d'intégrer les pays de 1'Est dans l'économie mondiale, ce qui est considéré comme le meilleur moyen de faire accéder ces pays à la prospérité économique ${ }^{2}$. Le soutien de la Suisse au processus de transition engagé dans ces pays comprend les axes suivants : la promotion d'un développement économique et social durable, conforme aux principes de l'économie de marché, la protection de l'environnement et l'utilisation rationnelle des ressources naturelles, la modernisation de l'infrastructure (production d'énergie par exemple).

Dans les lignes directrices Nord-Sud, l'un des quatre objectifs du Conseil fédéral portait sur la promotion de la prospérité commune (amélioration des conditionscadres pour un développement durable des pays en développement), sur la promotion du secteur privé dans les pays en développement, et sur la nécessité de faciliter l'accès aux marchés du Nord pour les produits des pays en développement.

Dans le nouveau rapport sur la politique extérieure $2000^{3}$, l'un des cinq objectifs de politique extérieure s'intitule «Sauvegarde des intérêts de l'économie suisse à $1^{\prime}$ 'étranger ${ }^{4}$. Les points forts thématiques comprennent la promotion des exportations des petites et moyennes entreprises (PME). En Europe centrale, le Conseil fédéral se concentrera au cours des années à venir sur la promotion des

1. Voir notamment les «Messages concernant la continuation du financement des mesures de politique économique et commerciale». Le Message du Conseil fédéral demande au Parlement d'adopter un crédit-cadre de dépenses pour trois ou quatre ans, le texte permet de faire le bilan des actions passées et de donner les grands axes de coopération pour les années à venir (le Message du 29 mai 1996 est le dernier message paru, le prochain est en préparation en 2001-2002).

2. «Message concernant l'arrêté fédéral sur la coopération avec les Etats de l'Europe de l'Est, du 19 septembre $1994 »$, Berne, in Feuille fédérale 1994 pp. 537-565 (message n 94.083).

3. Rapport sur la politique extérieure 2000. Présence et coopération: la sauvegarde des intérêts dans un monde en cours d'intégration, 15.11.2000.

4. Il est intéressant de relever le glissement de la formulation, puisque le rapport sur la politique extérieure dans les années 90 parlait de «l'accroissement de la prospérité commune», objectif qui devient dans le rapport 2000 «sauvegarde des intérêts de l'économie suisse à l'étranger». 
exportations et des investissements, ainsi que sur le soutien des marchés financiers locaux. Le rapport relève l'intérêt des instruments nouvellement créés en vue de soutenir des partenariats à long terme entre des entreprises suisses et des entreprises de pays en développement ou de pays en transition.

[D] ASTM 2001, partie Revue, sous-chapitre 6.1., pour la vue d'ensemble des instruments de politique économique extérieure, et sous-chapitre 5.1. pour le «Rapport sur la politique extérieure 2000 ».

Le Comité d'aide au développement (CAD) de l'OCDE a aussi à plusieurs reprises donné des orientations générales de la politique à adopter dans le domaine du soutien au secteur privé dans les pays en développement et les pays en transition $^{5}$. L'apport de capitaux étrangers, notamment les flux privés d'investissements directs, représente une contribution essentielle au financement du développement ${ }^{6}$.

\section{$\square$ Mise sur pied d'instruments novateurs de promotion des investissements}

Le Seco a développé, dans le cadre du $5^{\text {e }}$ crédit-cadre sur les mesures de politique économique et commerciale, une série d'instruments novateurs visant à stimuler les investissements suisses dans les pays en développement et les pays de l'Europe de l'Est'. Un nouvel accent a été mis sur le crédit de programme pour des appuis à l'investissement dans ces pays. Plusieurs instruments traditionnels de coopération (comme les financements mixtes) et la Garantie contre les risques à l'exportation (GRE, non comprise dans l'aide publique au développement) permettaient déjà de soutenir et d'encourager les exportations d'entreprises suisses vers ces pays. Dans le nouveau crédit-cadre, le Seco cherchait à développer un partenariat entre la Confédération et le secteur privé pour encourager plus spécifiquement les investissements à plus long terme et le transfert de technologies. L'idée est vraiment de trouver des synergies entre coopération au développement et investissements privés et la coopération peut jouer un rôle de catalyseur.

Sur le montant total du crédit-cadre de 960 millions de francs, donnant l'enveloppe générale des dépenses réparties sur quatre ans au minimum, 180 millions de francs étaient prévus pour des mesures de promotion des investissements. En 1999, les dépenses totales de l'aide publique gérée par le Seco se sont élevées à 146,5 millions de francs, dont 19,8 millions pour les instruments de promotion des investissements ${ }^{8}$. Les principaux pays bénéficiaires en 1999 ont été (par ordre décroissant d'importance) la Chine, la Colombie, le Maroc, le Vietnam et la Bolivie.

5. Voir par exemple:

OCDE, Echanges, investissements et développement. Pour la cohérence des politiques. Paris, OCDE, 1999, 90 p.

OECD, DAC (Comité d'aide au développement), DAC Orientations for Development Co-operation in Support of Private Sector Development, Paris, 3.6.1994 (note du Secrétariat du CAD).

6. Voir dans la partie Statistiques de cet Annuaire, tableau 2.1.B sur les flux financiers destinés aux pays en développement, ainsi que le tableau 2.4, sur les investissements directs suisses à l'étranger.

7. Voir: «Message concernant la continuation du financement et la réorientation des mesures de politique économique et commerciale au titre de la coopération au développement», du 29.5.1996 (message $\mathrm{n}^{\circ}$ 96.044). Le contenu du message a été détaillé dans l'Annuaire Suisse-Tiers Monde 1997 (pp. 152164). Voir aussi l'article de Laurent Guye dans la partie Analyses et positions de l'Annuaire SuisseTiers Monde 1998 «Coopération au développement: Pour un vrai partenariat entre la Confédération et le secteur privé » (pp. 117 à 124).

8. Source: Service statistique de la DDC. 
Le Seco participe actuellement à une large palette d'une vingtaine d'instruments de promotion des investissements.

«Les mesures ont été conçues de façon à couvrir les besoins des investisseurs aux différentes étapes que suit un projet, depuis la première ébauche jusqu'à sa réalisation. Ces différentes phases, ainsi que les instruments qui y correspondent, sont présentées sur le schéma suivant:

口 à l'étape de la formulation du projet, la facilité de participation aux frais d'étude permet à l'investisseur d'obtenir du Seco une avance couvrant jusqu'à $50 \%$ des coûts entraînés par une étude de faisabilité ou la réalisation d'une expérience pilote;

$\checkmark$ une fois le projet formulé, l'investisseur devra peut-être identifier un partenaire, dans un pays en développement ou en transition, qui réunisse les conditions techniques et de crédibilité nécessaires. La «Swiss Organization for Facilitating Investments » (SOFI), avec son vaste réseau d'organisations partenaires, pourra l'aider à le sélectionner et à négocier un accord de joint-venture (ou de toute autre forme de partenariat) mutuellement profitable;

a parvenu à l'étape de la réalisation du projet, l'investisseur aura peut-être besoin d'une source de financement, particulièrement en capital propre. La «Swiss Development Finance Corporation» (SDFC) ou l'un des fonds de partenariat ou de capital-risque auxquels participe le Seco pourra apporter une partie du financement requis et fournir les services d'ingénierie financière permettant de boucler l'ensemble du financement.

Un effort particulier d'information est consenti pour les projets d'investissement visant à introduire des technologies respectueuses de l'environnement. Les Centres de production propre (CPC) offrent aux entrepreneurs des pays en développement une information à jour et indépendante sur les technologies qui, pour les secteurs industriels qui sont les sources majeures de pollution, rendent compatibles l'objectif de la croissance et celui de la protection de l'environnement. Ils facilitent les contacts avec les détenteurs de ces technologies - en Suisse ou dans d'autres pays de l'OCDE - et renseignent également les PME sur les différentes possibilités de financement disponibles dans le pays concerné pour ce type d'investissement. » ${ }^{9}$

Le schéma suivant donne la vue d'ensemble des instruments développés ou cofinancés par le Seco. Le fonctionnement du Fonds pour le financement d'études, de la SOFI, de la Swiss Development Finance Corporation et des Cleaner Production Centers est examiné plus en détail dans ce dossier, avec des textes de présentation et un premier bilan de leur fonctionnement lors de l'atelier organisé à Olten. Les autres instruments sont évoqués dans la partie «Description des instruments de promotion des investissements du Seco». Nous nous concentrons volontairement dans ce dossier sur l'aide bilatérale, et ne parlons donc pas de la participation de la Suisse à des organisations internationales ou à des fonds internationaux qui visent aussi la promotion des investissements dans les pays en développement (Société financière internationale - SFI, filiale de la Banque mondiale, l'Agence multilatérale de garantie des investissements - AMGI,

9. Seco www.seco-admin.ch. Présentation «Investissements dans les pays en développement ou en transition». 
Banques régionales de développement et divers fonds fiduciaires liés à ces banques (trust funds), servant par exemple à financer des études de faisabilité de certains projets).

\section{Schéma 1: Vue d'ensemble des instruments de promotion des investissements Phases d'un projet d'investissement}

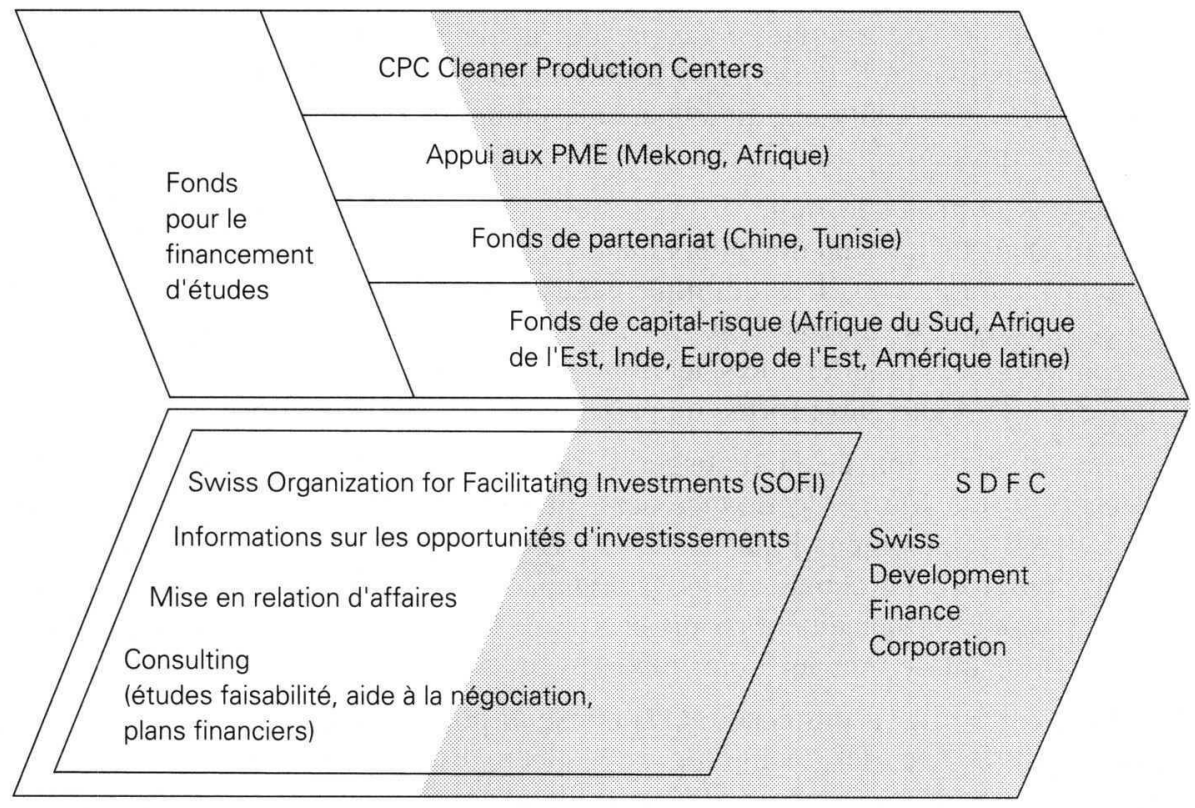

Préparation de l'investissement / Réalisation 


\section{SOFI - SWISS ORGANIZATION FOR FACILITATING INVESTMENTS}

\section{ANDREAS RAGAZ*}

La Swiss Organization for Facilitating Investments (SOFI) a été créée en 1997 par l'OFAEE, l'actuel Seco. La SOFI est ainsi un organisme gouvernemental, mais géré sous mandat par KPMG Switzerland. Elle a son siège à Zurich (Stauffacherstrasse 45) et occupe actuellement 16 personnes.

Sa raison d'être: apporter de l'aide aux entreprises suisses qui investissent dans des pays en développement et en transition. La SOFI collecte et transmet des informations, établit des contacts, recherche des partenaires commerciaux appropriés, contribue à trouver des sources de financement, aide à structurer des projets et des plans d'activité, donne des conseils généraux en matière de projets d'investissement. Elle organise par ailleurs des séminaires, des conférences et autres manifestations consacrés aux investissements dans des pays en développement et en transition. La SOFI réalise également des programmes de formation dans ces pays.

Son fonds d'études a pour objet de prendre en charge jusqu'à la moitié des frais d'études et de projets pilotes pour les entreprises suisses qui envisagent d'investir concrètement dans certains pays en transition ou en développement. Si les résultats de ces études ou essais montrent que l'investissement projeté serait irréalisable ou insuffisamment rentable, conduisant ainsi à y renoncer, le crédit en question pourra être transformé en donation. Si le projet aboutit, le crédit sera remboursable avec les intérêts.

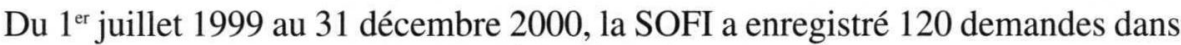
ce sens, et 52 projets concrets ont été présentés avec une requête de financement par le fonds d'études.

Critères de sélection:

évaluation provisoire de la faisabilité économique du projet d'investissement (p. ex. risques et rentabilité probables);

- effet de développement attendu (p. ex. contribution au développement économique du pays partenaire, transfert de technologie et de savoir-faire, aspects sociaux et écologiques);

- crédibilité des investisseurs: existence d'investisseurs prêts à s'engager si l'étude ou le projet pilote donne des résultats probants;

- rapport entre le coût de l'étude ou du projet pilote et le montant de l'investissement prévu;

- qualité du plan d'exécution de l'étude ou du projet pilote;

- qualifications des personnes responsables de l'étude ou du projet pilote.

* Andreas Ragaz est employé de banque diplômé. Parallèlement à une carrière conventionnelle dans le secteur bancaire et après celle-ci, il a été membre fondateur de l'Alternative Bank ABS; il a dirigé l'ABS de 1990 à 1994. De 1996 à 1999, il a conseillé des banques et formé des spécialistes dans cette branche en Moldavie et en Ukraine. Andreas Ragaz est responsable du fonds d'études du Seco à la SOFI depuis le $1^{\text {er }}$ juillet 1999. 
Il y a eu 23 demandes de financement présentées à la commission d'experts, dont 18 ont donné lieu à une recommandation positive et 5 ont été rejetées. Au 31 décembre 2000, le fonds d'études comportait 26 crédits totalisant quelque 7 millions de francs. La SOFI publie sur Internet une liste des projets, sans indiquer les noms.

Tableau 1: Projets approuvés au 31.12.2000 par régions (en francs)

\begin{tabular}{lrrr}
\hline & Nb de projets & Montant & Part du total \\
\hline Chine & 4 & $1^{\prime} 01^{\prime} 225$ & $14,3 \%$ \\
\hline Inde & 4 & $669^{\prime} 564$ & $9,5 \%$ \\
\hline Asie, autres & 4 & $1^{\prime} 426^{\prime} 500$ & $20,3 \%$ \\
\hline \multicolumn{1}{c}{ Sous-total Asie } & 12 & $3^{\prime} 097^{\prime} 289$ & $44,1 \%$ \\
\hline Afrique du Nord & 2 & $700^{\prime} 000$ & $10,0 \%$ \\
\hline Afrique subsaharienne & 4 & $1^{\prime} 000^{\prime} 345$ & $14,2 \%$ \\
\hline Sous-total Afrique & 6 & $1^{\prime} 700^{\prime} 345$ & $24,2 \%$ \\
\hline Amérique latine & 1 & $50^{\prime} 000$ & $0,7 \%$ \\
\hline Europe sans CEl & 3 & $858^{\prime} 600$ & $12,2 \%$ \\
\hline CEl & 4 & $1^{\prime} 316^{\prime} 000$ & $18,8 \%$ \\
\hline Total & $\mathbf{2 6}$ & $\mathbf{7}^{\prime} \mathbf{0 2 2} \mathbf{2 3 4}$ & $\mathbf{1 0 0} \%$ \\
\hline
\end{tabular}

Tableau 2: Projets approuvés au 31.12.2000 par branches (en francs)

\begin{tabular}{lrrr}
\hline & Nb de projets & Montant & Part du total \\
\hline Agriculture, produits alimentaires & 6 & $1^{\prime} 906^{\prime} 000$ & $27,1 \%$ \\
\hline Boissons & 1 & $320^{\prime} 000$ & $4,6 \%$ \\
\hline Bois & 1 & $229^{\prime} 800$ & $3,3 \%$ \\
\hline Construction & 2 & $318^{\prime} 100$ & $4,5 \%$ \\
\hline Textile & 2 & $325^{\prime} 064$ & $4,6 \%$ \\
\hline Papier & 1 & $635^{\prime} 000$ & $9,0 \%$ \\
\hline Métaux & 1 & $460^{\prime} 000$ & $6,6 \%$ \\
\hline Chimie, plastique & 2 & $241^{\prime} 345$ & $3,4 \%$ \\
\hline Produits semi-finis & 1 & $115^{\prime} 125$ & $1,6 \%$ \\
\hline Electronique & 2 & $500^{\prime} 000$ & $7,1 \%$ \\
\hline Electricité, gaz, eau & 3 & $777^{\prime} 800$ & $11,1 \%$ \\
\hline Poste, téléphone & 2 & $750^{\prime} 000$ & $10,7 \%$ \\
\hline Transports & 2 & $444^{\prime} 000$ & $6,3 \%$ \\
\hline Total & $\mathbf{2 6}$ & $\mathbf{7}^{\prime} \mathbf{0 2 2} \mathbf{2 3 4}$ & $\mathbf{1 0 0} \%$ \\
\hline \multicolumn{1}{c}{ dont technologies environnement. } & 8 & $1^{\prime} 891^{\prime} 745$ & $26,9 \%$ \\
\hline dont nouvelle économie & 2 & $750^{\prime} 000$ & $10,7 \%$ \\
\hline
\end{tabular}

Tableau 3: Projets approuvés au 31.12.2000 par taille des entreprises (en francs)

\begin{tabular}{lcrr}
\hline & Nb de projets & Montant & Part du total \\
\hline Micro (< 10 personnes) & 12 & $2^{\prime} 194^{\prime} 934$ & $31,2 \%$ \\
\hline Petites (10-49 personnes) & 5 & $1^{\prime} 717^{\prime} 800$ & $24,5 \%$ \\
\hline Moyennes (50-249 personnes) & 8 & $2^{\prime} 729^{\prime} 500$ & $38,9 \%$ \\
\hline Grandes (dès 250 personnes) & 1 & $380^{\prime} 000$ & $5,4 \%$ \\
\hline Total & $\mathbf{2 6}$ & $\mathbf{7}^{\prime} \mathbf{0 2 2} \mathbf{2 3 4}$ & $\mathbf{1 0 0 \%}$ \\
\hline
\end{tabular}

On ne mesure pas concrètement les effets produits sur le développement. La SOFI demande aux entreprises des rapports réguliers sur l'évolution de leur projet jusqu'au moment où le crédit est remboursé. Le projet est évalué avant que l'on approuve le crédit, puis surveillé pendant et après son versement. Souvent, la SOFI est mandatée pour accompagner les projets et fait alors des visites sur 
place. Dans ce cas, une de ses principales fonctions est d'établir des contacts avec les autorités et les milieux professionnels. La SOFI a signé des accords de coopération avec des autorités et des associations professionnelles dans 55 pays.

\section{Projets financés par le fonds d'études}

\begin{tabular}{ll}
\hline Pays & Description du projet \\
\hline Vietnam & Projet pilote de plantations de framboises. \\
& Etude de faisabilité relative à des services sur Internet. \\
& Etude de marché et projet pilote concernant la transformation de viande et la \\
& fabrication de saucisses. \\
\hline Inde & Examen de la possibilité d'aménager dans le Tamil Nadu un projet pilote et un \\
& centre de formation destinés à améliorer la filature. \\
& Etude de faisabilité et projet pilote pour la réalisation d'un système de transport \\
& combiné par containers. \\
& Etude de faisabilité concernant une entreprise en participation pour la fabrication \\
& et la modernisation de bogies pour Indian Railways. \\
& Etude de marché et projet pilote pour la fabrication de réactifs et de tests san- \\
& guins pour le diagnostic clinique.
\end{tabular}

Pérou

Etude de faisabilité avec installation pilote concernant une entreprise en participation pour la fabrication de cellules photovoltaïques.

Chine

Projet pilote pour la fabrication de billes de stylo.

Etude d'un procédé de percement de conduites d'adduction d'eau destiné à en limiter l'impact écologique.

Etude de faisabilité avec projet pilote pour le traitement des eaux résiduaires de la fabrication du caoutchouc et leur valorisation pour la production d'énergie, d'engrais, d'aliments pour poissons.

Afrique du Sud Etude portant sur une technologie d'absorption d'huile.

Côte d'Ivoire Projet pilote pour la production de tomates hors sol en serres.

Projet pilote pour la production de plantes d'appartement en hydroculture selon les principes de la Pl, et leur exportation en Europe.

Projet pilote pour la production de papayes.

Tunisie Projet pilote de panneaux publicitaires photovoltaiqques à l'aéroport de Tunis.

Egypte Projet pilote relatif à des services dans les télécommunications.

Géorgie Etude de marché et projet pilote pour la mise en bouteilles d'eau minérale.

Russie Projet pilote pour la modernisation d'unités centrales de systèmes de chauffage et d'eau chaude.

Projet pilote pour le conditionnement de poisson (sandre) selon les critères occidentaux aux fins d'exportation en Europe et aux Etats-Unis. 


\begin{tabular}{ll}
\hline Pays & Description du projet \\
\hline Turkménistan & Projet pilote pour un procédé moderne et non polluant de teinture de chaussettes. \\
\hline Burkina Faso & $\begin{array}{l}\text { Projet pilote pour la production de vinaigre de mangue selon les critères occiden- } \\
\text { taux et sa vente en Suisse. }\end{array}$ \\
\hline Pologne & Etude de marché et projet pilote pour la fabrication de chaudières à bois. \\
\hline Bulgarie & Projet pilote pour l'exploitation de bois de hêtre. \\
\hline $\begin{array}{l}\text { Roumanie } \\
\text { Chine, Inde, }\end{array}$ & $\begin{array}{l}\text { Phase pilote d'un projet de métallurgie. } \\
\text { Amérique latine }\end{array}$ \\
\hline
\end{tabular}

\title{
CPC, CLEANER PRODUCTION CENTERS
}

\author{
Hans-Peter EgleR*
}

\section{CONTEXTE}

Un des effets de la mondialisation est d'accroître la demande de technologies respectueuses de l'environnement - dans les pays en développement et en transition comme partout ailleurs. Un certain nombre de facteurs stimulent cette demande: les pays importateurs appliquent des normes écologiques plus sévères pour les produits industriels; la politique environnementale des pays en développement et en transition s'oriente de plus en plus sur des critères commerciaux ; consommateurs, habitants, groupes d'intérêts et marchés financiers se mettent à évaluer systématiquement le comportement écologique des entreprises.

La Suisse jouit d'une expérience et d'un savoir-faire considérables dans le domaine des technologies environnementales (recherche et développement) aussi bien qu'au niveau des normes. Beaucoup de compétences s'y sont développées depuis les années 60 dans les domaines de l'assainissement (solutions en fin de cycle ou end of pipe), de la récupération (recyclage) et enfin de la limitation des pollutions par des méthodes de production plus efficaces et plus propres. L'industrie suisse a réalisé des produits et des services de réputation internationale dans le domaine de 1'environnement. Mais ces prestations - en particulier celles qui ont été élaborées par des PME - sont (encore trop) rarement proposées dans les pays en développement et en transition, notamment à cause des frais de transaction élevés que cela implique pour les fournisseurs comme pour les clients potentiels.

Le Seco s'est par conséquent donné pour objectif de mettre en place dans certains pays partenaires de la Suisse un instrument complet et cohérent pour le

* Licencié. ès sciences, Seco. 
transfert de technologies propres. Ce projet se concrétise sous la forme de centres de technologies respectueuses de l'environnement (cleaner production centers, CPC).

\section{$\square$ Terminologie}

La stratégie des CPC est destinée à donner aux fournisseurs de biens et de services les moyens de ménager les ressources en produisant de façon à la fois plus économique et plus écologique. Elle vise à résoudre les problèmes à la source, au niveau des causes, plutôt que de se contenter d'éliminer les déchets à la fin du processus de production (intervention au point de rejet, solution end of pipe). Outre l'amélioration des procédés, cette approche englobe l'optimisation des produits à réaliser, l'analyse de leurs cycles de vie, l'instauration de systèmes de management environnemental (SME, ISO 14000), etc. Les CPC constituent ainsi une application méthodique du principe selon lequel les secteurs, les entreprises et les processus de production doivent être performants pour rester compétitifs sur le marché international. L'idée est de passer systématiquement en revue les moyens d'optimiser la transformation des ressources énergétiques et des matières afin de réduire les prix de revient des produits et des services tout en ménageant l'environnement (moins de déchets et d'émissions par unité de production). La protection de l'environnement constitue ainsi un effet secondaire bienvenu des CPC, l'objectif principal étant une rentabilité accrue.

\section{$\square$ Fonction des CPC}

Toute technique environnementale appliquée à la fin du processus de fabrication entraîne des frais supplémentaires sans apporter aucun gain d'efficacité. L'entreprise située dans un pays en développement ou en transition qui se contente de protéger l'environnement par assainissement en fin de cycle (stratégie end of pipe) risque ainsi une perte de compétitivité par rapport à l'entreprise qui ménage les ressources, car son manque de dynamisme l'empêchera probablement d'accéder au marché mondial (voir ISO 14000).

L'optimisation systématique des processus de production débouche sur des produits de meilleure qualité et rend l'entreprise plus performante. De plus, les économies (d'énergie, de matières) réalisées grâce aux CPC permettront à l'entreprise d'investir dans les techniques environnementales alors qu'elle n'en aurait pas eu les moyens autrement. Dans les pays pauvres notamment, CPC et technologies de fin de cycle ne sont pas antagonistes: au contraire, il serait illusoire de penser que des entreprises pourraient acquérir des systèmes d'assainissement d'une certaine ampleur avant d'avoir optimisé leur production par les CPC.

Des méthodes de production plus respectueuses de l'environnement impliquent aussi des postes de travail plus sûrs (prévention des accidents majeurs, normes de santé au travail). Une compétitivité accrue se traduit par des emplois plus nombreux et meilleurs. Et les conditions de vie dans la région ne peuvent que profiter d'une réduction des quantités de déchets et d'eaux usées.

Les CPC pratiquées dans des pays en développement et en transition favorisent, selon le point de vue suisse, l'exportation de technologies environnementales en réduisant les frais d'information et de transaction, surtout pour les PME. En effet, le travail effectué dans ce domaine fait connaître les situations locales et permet alors d'élaborer des offres mieux ciblées, de mieux cerner les besoins à faire 
connaître aux producteurs de ces technologies dans les pays industrialisés (dont la Suisse). Un CPC peut ainsi contribuer à réduire les frais de transaction du fournisseur de technologie.

\section{$\square$ Objectif}

Le but que visent les CPC appuyés par le Seco est de contribuer dans une large mesure à l'amélioration des techniques de production et à l'instauration de méthodes de fabrication plus écologiques dans les pays en développement et en transition. Pour ce faire, les CPC établis dans ces pays offrent - à des conditions de rémunération variables selon les cas - une vaste gamme de services dans le domaine des technologies à faible impact sur l'environnement. Ces centres conseillent les entreprises en matière de gestion environnementale et de rendement énergétique, pour le choix d'équipements et de systèmes peu polluants, mais aussi pour les technologies d'assainissement en fin de cycle (end of pipe technologies) et la manière de les financer. Le conseil des collectivités porte essentiellement sur la gestion des déchets.

Les CPC jouent le rôle d'intermédiaires entre les industries nationales et internationales et, le cas échéant, les collectivités. Ils coopèrent avec des instances nationales spécialisées (associations professionnelles, experts, universités, etc.) et avec des institutions techniques en Suisse (centres de référence). Leur fonction consiste ainsi, d'une certaine manière, à préparer le marché.

Leurs clients sont surtout des PME confrontées à des pressions croissantes pour qu'elles réduisent leurs atteintes à l'environnement, mais ne disposant pas du même savoir-faire que les grandes entreprises dans le domaine des technologies propres.

\section{Schéma 2: Exemple d'établissement d'un CPC en Colombie}

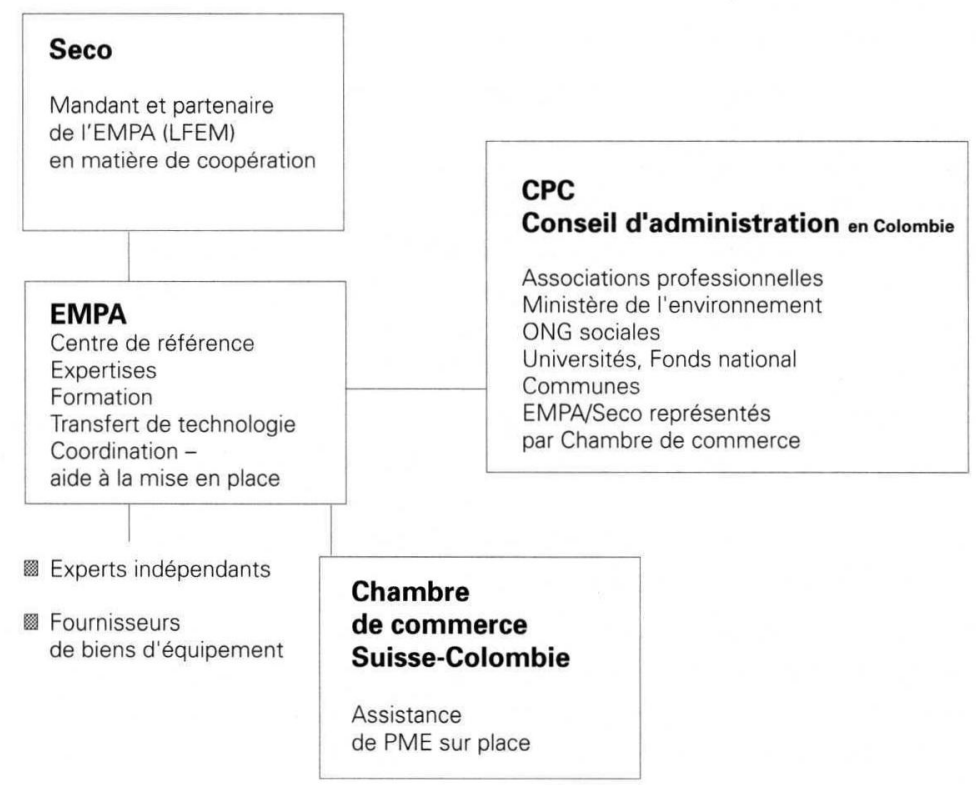




\section{$\square$ Intérêt pour la politique de développement}

Des CPC ont été créés en Amérique centrale, en Colombie, au Maroc, au Vietnam; d'autres sont en préparation en Inde, au Brésil, au Pérou et en Chine. La coordination des projets sur place se fait en partie sur le mode bilatéral, en partie par l'ONUDI à Vienne ou avec d'autres donateurs ou institutions de financement internationales. D'autres donateurs bilatéraux soutiennent des projets analogues.

Afin de pouvoir mesurer l'effet produit par ces CTRE, le Seco a élaboré avec leur collaboration douze indicateurs d'impact à évaluer deux fois par année, cela à l'intention des CTRE eux-mêmes, des centres de référence et des organisations partenaires (ONUDI).

Leurs centres de référence en Suisse mettent les CTRE en contact avec divers organismes spécialisés (experts, institutions de formation et de mesure, producteurs de biens d'équipement, etc.). Les CTRE sont par ailleurs reliés entre eux.

\section{$\square$ Intérêt pour l'économie suisse}

En ce qui concerne les transferts de biens d'équipement, les expériences faites jusqu'à présent restent en deçà des attentes; cela s'explique sans doute par le fait que les CTRE n'ont pas encore atteint un niveau suffisant de maturité institutionnelle (le premier a été ouvert au début de 1998). Il faut par ailleurs du temps pour mettre en place dans le pays partenaire une filière de projets intéressants pour les PME suisses.

Les CTRE sont des institutions «indirectes» qui travaillent le plus souvent avec le secteur privé (experts locaux et internationaux) et offrent toute la gamme des services. Il en résulte une valeur ajoutée et les experts locaux bénéficient en même temps d'un transfert de know-how. Cela renforce les acteurs économiques sans faire concurrence au marché libre.

\section{SDFC, SOCIÉTÉ FINANCIÈRE SUISSE POUR LE DÉVELOPPEMENT}

\section{Monika Egger et Susanne Grossmann*}

La SDFC ${ }^{10}$ est une société anonyme dont le capital de 100 millions de francs est souscrit à $49 \%$ par la Confédération et à $51 \%$ par des actionnaires privés (état du capital à fin 2000: 50 millions de francs). Son mode de fonctionnement est analogue à celui de la Société financière internationale (SFI), filiale de la Banque mondiale qui aide le secteur privé des pays en développement en lui proposant des services financiers. La société doit s'autofinancer et l'Etat modère ses directives en matière d'investissements afin de ne pas compromettre sa viabilité. La définition des critères qui régissent le soutien de projets incombe au Conseil d'administration de la SDFC. Celui-ci comprend quatre représentants de l'économie privée et trois représentants de la Confédération. L'article 27 des statuts

* Suzanne Grossmann est responsable de la coopération avec l'Afrique au sein du secteur Promotion des investissements du centre de prestations Développement et transition du Seco.

10. Présentation de la Société financière suisse pour le développement ou Swiss Development Finance Corporation (SDFC) dans l'Annuaire Suisse-Tiers monde 1997, p. 160. 
stipule l'obligation pour le Conseil d'administration d'élaborer une charte prescrivant que les opérations de la société doivent être régies par des critères d'équilibre économique, social et écologique. La SDFC a accès à d'autres instruments de promotion de la Confédération, notamment au fonds d'études pour les questions de financement. Son but est d'encourager et d'établir des partenariats durables entre des sociétés de pays appartenant à l'OCDE - en priorité de la Suisse - et des entreprises situées dans des pays en développement ou en Europe centrale et orientale ainsi que dans la CEI. Ses principaux instruments: participations, prêts et garanties. La SDFC finance au maximum $25 \%$ du coût global d'un projet. Entrent en considération, comme bénéficiaires, les pays dont le revenu par habitant est inférieur à 5000 dollars, donc surtout les pays à revenus intermédiaires où les investisseurs étrangers (suisses) hésitent encore à s'aventurer seuls. Il est prévu de procéder après cinq ans à une évaluation a posteriori de l'effet de développement, au moyen d'un questionnaire inspiré de celui utilisé par la SFI. Cette analyse devra examiner en particulier les aspects suivants: viabilité économique de l'entreprise, transfert de technologie, formation, impact du projet sur le marché intérieur, répercussions sur l'environnement, incidences sociales.

La SDFC constitue un instrument spécial, qui se situe à mi-chemin entre la promotion économique suisse et la coopération au développement. Cette société est devenue opérationnelle en mars 1999, après une longue phase de préparation et de mise en place. Son capital atteint actuellement (octobre 2000) 50 millions de francs. La «participation minoritaire» de la Confédération (49\%) est inhabituelle par rapport à d'autres pays où l'Etat est majoritaire dans ce genre de société. La Confédération entend ainsi responsabiliser les investisseurs suisses, tout en ayant elle-même une fonction d'investisseur et en prenant à sa charge le risque politique de ces opérations. Cette prise en charge du risque politique est une concession à l'économie privée, laquelle a consenti en contrepartie que le siège de la SDFC soit en Suisse (et non pas dans un paradis fiscal). De plus, la Confédération assume une partie des frais inhérents, notamment, à l'encouragement de petits projets. 


\subsection{NOUVEAUX INSTRUMENTS DU SECO DESTINÉS À PROMOUVOIR LE DÉVELOPPEMENT: PREMIĖRES OBSERVATIONS}

\section{SYNTHÈSE DE LA TABLE RONDE}

\section{Monika Egger}

La table ronde s'est déroulée le 26 octobre 2000 et a été subdivisée comme suit: présentation des participant-e-s et de leurs activités en rapport avec les instruments de promotion, présentation des différents instruments par le Seco, débat sur les questions soulevées. Les participant-e-s:

Peter Bosshard: collaborateur de la Déclaration de Berne (DB). Responsable du programme «Relations financières Nord-Sud» avec un intérêt particulier pour les flux de capitaux privés. La Déclaration de Berne a exprimé ses préoccupations relatives aux nouveaux instruments lors de l'élaboration du message concernant le cinquième crédit de programme.

Thomas Bürki: propriétaire d'un bureau d'ingénieurs-conseils dans les domaines suivants: énergie, écologie et politique. Consultant en matière de Cleaner Production Centers (CPC, centres de production propre).

Hans-Peter Egler: suppléant du chef du secteur Coopération pour le commerce et la technique environnementale au Seco. Depuis 1994, collaborateur du centre de prestations Développement et transition, responsable de l'application des aspects commerciaux des conventions internationales dans les pays en développement et en transition, notamment pour les Cleaner Production Centers.

Martina Gebhart: nouvelle collaboratrice du Seco dans le domaine des Cleaner Production Centers.

Susanne Grossmann: collaboratrice du Seco depuis 1995, elle travaille depuis janvier 1999 au sein du centre de prestations Développement et transition. Responsable de la coopération avec l'Afrique pour le secteur Promotion des investissements.

Urs Heierli : collaborateur de la section Emploi et revenu de la DDC. Responsable pour le marketing, la production agricole et l'économie, ainsi que pour la promotion du secteur privé.

Nadine Keim: collaboratrice de la Communauté de travail Swissaid, Action de Carême, Pain pour le prochain, Helvetas, Caritas, Lausanne. Responsable du domaine relations commerciales internationales et OMC.

Fredy von Niederhäusern: directeur de ASICO SA, à Bioggio. Avec le soutien de la SOFI, ASICO SA a créé en Chine une entreprise qui produit des billes pour les stylos à bille et qui compte cinquante employé-e-s.

Gérard Perroulaz: membre du comité de rédaction de l'Annuaire Suisse-Tiers Monde et chargé de recherche à l'IUED, Genève.

Andreas Ragaz: collaborateur de la SOFI, responsable du fonds d'études (FFPIS).

Monika Egger: économiste, consultante dans le domaine de l'économie et du développement, membre du comité de rédaction de l'Annuaire Suisse-Tiers Monde. Animatrice de la table ronde et auteur du rapport de synthèse.

Monika Zehe : traductrice; retranscription des débats. 


\section{PRÉSENTATION DES NOUVEAUX INSTRUMENTS DE PROMOTION}

Dans son message demandant l'adoption du $5^{\mathrm{e}}$ crédit de programme destiné à financer des mesures de politique économique et commerciale ${ }^{11}$, entré en vigueur au début de 1997, le Conseil fédéral propose une réorientation de ces mesures ainsi qu'une série de nouveaux instruments de promotion ${ }^{12}$ :

\section{$\square$ La SOFI (Swiss Organization for Facilitating Investments)} et le fonds d'études (présentation: Andreas Ragaz)

La SOFI a pour objectif d'apporter un appui aux entreprises suisses qui investissent dans les pays en transition et en développement. Ses activités comprennent l'organisation de manifestations, la diffusion d'informations, la création de contacts, des conseils (gestion et financement) et la recherche de PME suisses, prêtes à investir dans les pays en développement, ainsi que de partenaires appropriés sur place pour assurer la réalisation de projets. Sa tâche principale, mais aussi la plus difficile, consiste à trouver des moyens financiers pour les projets qui lui sont soumis.

En tant que service de consultation et de prise en charge neutre, soutenue par le gouvernement suisse, la SOFI joue un rôle important en appuyant les entreprises locales et suisses dans leurs négociations avec les bailleurs de fonds et avec les banques. Les prestations de base de la SOFI sont en principe gratuites pour les clients, mais les consultations plus approfondies et un appui actif sur place font l'objet de mandats rémunérés. A la fin de l'an 2000, la SOFI avait signé plus de cent protocoles d'accord avec des agences privées et gouvernementales de promotion des investissements et avec des associations d'entreprises privées.

Le fonds pour le financement d'études de faisabilité et de projets pilotes pour des programmes d'investissement (fonds d'études, FFPIS) a également été créé en 1997 et fait aussi partie de l'offre de base de la SOFI. Il intervient tout au début d'un projet d'investissement et sert à financer des études de faisabilité ainsi que des projets pilotes destinés à évaluer la rentabilité et la faisabilité technique des projets envisagés. Les projets doivent en effet être viables du point de vue commercial. Par la suite, au stade de la réalisation du projet (investissements effectifs), d'autres instruments de financement entrent en jeu: la SFSD, par exemple, ou des fonds bilatéraux et régionaux, tels que les fonds suisses de partenariat avec la Chine ou avec la Tunisie, qui accordent des prêts de capital-risque.

En 1999, le fonds d'études a transmis neuf projets à la SOFI pour réalisation et a financé, en 2000 , les études préalables à dix-neuf autres projets. Les premières expériences du fonctionnement de ce fonds montrent que la plupart des projets connaissent une évolution positive. Il apparaît cependant que le passage de la phase d'investigation à la réalisation, avec les investissements requis, pose souvent problème. Les PME peinent à trouver les moyens nécessaires pour financer cette phase de mise en place et de démarrage - qui exige beaucoup de temps et

11. «Message concernant la continuation du financement et la réorientation des mesures de politique économique et commerciale au titre de la coopération au développement », 29.5.1996, Berne.

12. Lire la présentation détaillée du message et des instruments, ainsi que les prises de position des milieux économiques, des œuvres d'entraide et des organisations de développement dans l'Annuaire Suisse-Tiers Monde 1997, p. 152 ss. 
de travail - car les investisseurs, telle la SFSD, jugent qu'il est trop tôt, à ce stade, pour juger de la rentabilité d'un projet.

Le fonds d'études applique les mêmes principes qu'un fonds de capital-risque mais ne finance que les phases d'investigation et de test d'un projet. Le Seco part donc de l'hypothèse que les intérêts engrangés par le fonds ne couvriront pas ses pertes. Le fonds est alimenté par le $5^{\mathrm{e}}$ crédit de programme et a pour but de stimuler le développement des pays partenaires. Voici les critères utilisés pour évaluer l'impact sur le développement: création d'emplois, transfert de connaissances, transfert de technologies et prise en compte d'aspects sociaux. En matière d'environnement, l'exigence minimale est que les projets ne portent pas atteinte à l'environnement.

A la fin de l'an 2000, la SOFI comptait seize collaborateurs et collaboratrices. La demande dont font l'objet ses prestations est «moyenne avec une tendance à la hausse» et le nombre des demandes provenant de Suisse romande est supérieur à la moyenne. La réserve des clients s'explique sans doute par la méconnaissance de la SOFI dans les milieux économiques visés.

\section{La Société financière suisse pour le développement (SFSD) $)^{13}$} et les fonds de capital-risque (présentation: Susanne Grossmann)

Dans le cadre de la coopération au développement, le Seco est chargé de promouvoir la croissance durable et de lutter contre la pauvreté dans les pays en développement et en transition. La palette des mesures disponibles comprend la promotion du secteur privé et des investissements. A l'aide de ces instruments, le Seco voudrait couvrir les différentes phases d'un projet d'investissement, de la phase de préinvestissement à la réalisation.

Le Seco détient des participations au sein de dix-neuf intermédiaires financiers divers, qui comprennent des fonds de leasing pour l'investissement, des fonds de garantie, des fonds de capital-risque (surtout en Afrique), des fonds sectoriels tels que les fonds d'infrastructure (des fonds qui mettent leurs fonds propres à disposition de projets d'infrastructure privés) et une série de fonds environnementaux, ainsi que des fonds de partenariat bilatéraux (avec la Chine et l'Inde notamment). Les moyens engagés dans ce domaine s'élèvent à 180 millions de francs, dont la majeure partie est gérée par la SDSF. Le Seco s'est fixé pour objectif de doter la SDSF d'un capital de 100 millions de francs, dont 49 millions seraient apportés par la Confédération.

A fin octobre 2000, trois projets d'investissement de la SDSF ont été approuvés. Ils concernent la Zambie, la Russie et le Brésil (NordEste). La SDSF ne se contente pas de participer à des financements, elle propose aussi des prestations complètes (élaboration de tout le paquet financier) et contribue surtout à trouver d'autres investisseurs. La participation de la SDSF à un projet est limitée à dix ans, mais peut être prolongée de deux ans supplémentaires. Passé ce délai, le capital de la SDSF doit être retiré du projet. Les investissements consentis dans les trois projets mentionnés ci-dessus, se situent entre 1 million et 5,5 millions de francs. L'investissement peut servir à créer une coentreprise, mais peut aussi se résumer à une participation au financement d'un fournisseur ou d'un acheteur. 
Les fonds de capital-risque exercent leur influence sur le développement à deux niveaux: au niveau de l'entreprise à laquelle ils offrent un accès direct à du capital-risque et au niveau du pays.

口 Au niveau de l'entreprise: L'entreprise concernée bénéficie non seulement d'un apport de capital, mais aussi du transfert de technologies et de savoirfaire (qui va de pair avec l'apport financier), ainsi que d'un appui assuré par l'administrateur du fonds, par exemple dans le domaine du marketing, de la structure financière et de la gestion du personnel. Cette combinaison de capital et de savoir-faire est un critère important, notamment lorsqu'il s'agit de soutenir des petites et moyennes entreprises. En effet, la direction du fonds intègre les PME bénéficiaires dans un réseau où elles peuvent conclure des partenariats fort utiles.

口 Au niveau du pays: L'effet positif sur le développement est lié au type d'investissement consenti. En effet, les fonds de capital-risque placent de l'argent dans des idées commerciales qui n'auraient guère de chance d'être réalisées sans l'apport de ces fonds. Il s'agit d'accroître la valeur des entreprises, ce qui se traduit souvent par la création d'emplois et/ou par une hausse de la qualification des emplois. Par ailleurs, ces investissements génèrent des avantages fiscaux non négligeables (augmentation des recettes fiscales). Les fonds de capitalrisque soutenus par le Seco ne viennent en aide qu'aux entreprises qui s'engagent à présenter régulièrement leur bilan. C'est une exigence difficile à faire comprendre dans certaines PME, surtout en Afrique. Il arrive souvent que les entreprises bénéficiaires soient des sociétés exportatrices, de sorte que les fonds de capital-risque contribuent grandement à la promotion des exportations.

Premiers enseignements tirés de la mise en œuvre des fonds de capital-risque :

$\checkmark$ Le succès d'un tel fonds dépend avant tout d'une bonne gestion et le facteur humain est primordial pour permettre aux entreprises bénéficiaires d'engranger un maximum de connaissances afin d'améliorer leur propre système de gestion. L'administration de ces fonds est confiée en priorité à des acteurs locaux qui possèdent le potentiel requis et qui sont à même d'acquérir les compétences de gestion nécessaires à condition de bénéficier d'un appui et d'une promotion adéquats. La nouvelle génération africaine, par exemple, comprend nombre de personnes au bénéfice d'une excellente formation qui sont à même d'assumer ce genre de tâche, surtout si on leur permet de suivre des cours de perfectionnement appropriés.

\ Le principal défi à relever est d'améliorer 1'accès des fonds de capital-risque pour les PME, surtout pour les petites entreprises. Celles-ci n'intéressent que rarement les fonds de capital-risque, car les coûts des transactions sont trop élevés. C'est pourquoi les responsables au sein du Seco se demandent s'il faut maintenir ces fonds dans le domaine des petites entreprises, ou s'il ne serait pas plus indiqué d'élaborer de nouveaux instruments. (Les investissements de moins de 100'000 dollars américains ne sont pas intéressants pour ce genre de fonds, car le suivi de l'entreprise représente un coût trop élevé par rapport à l'investissement en capital.)

\section{$\square$ Les Cleaner Production Centers (CPC) (présentation: Hans-Peter Egler)}

Les activités du Seco en matière de coopération au développement sont vastes et comprennent non seulement la promotion des investissements et du commerce, 
mais aussi le renforcement des règles du commerce international adoptées dans le cadre de l'OMC. D'autres conventions internationales ont par ailleurs un impact sur le commerce: par exemple les conventions internationales sur l'environnement. Dans ce domaine, le travail du Seco consiste à soutenir les pays en développement et en transition dans leurs efforts d'intégration sur le marché mondial et à promouvoir leurs propres compétences en vue de participer pleinement à la mise en place d'une économie mondiale globalisée.

Les Cleaner Production Centers sont un instrument que le Seco applique dans les domaines liés à l'environnement et qui ont une influence sur le commerce. Le long processus qu'implique l'application des conventions adoptées à Rio a mis en évidence les principaux besoins des pays en développement et en transition: développement de capacités, transfert de technologies et de savoir-faire, possibilité de disposer de ressources financières complémentaires. Le principal objectif consiste à renforcer et à promouvoir les capacités locales. On parle beaucoup du transfert de technologies environnementales depuis Rio, mais il est rare de le voir appliqué avec succès. Les CPC peuvent remédier à ces échecs.

Dans le cas des CPC, le Seco applique une approche institutionnelle. Il s'agit de créer des centres qui assurent le transfert de technologies et de techniques respectueuses de l'environnement, et qui favorisent ainsi l'adoption de modes de production non polluants par les entreprises locales. L'élimination des déchets, autrement dit leur gestion publique, est un autre domaine d'activité de ces centres.

Mais qu'entend-on en fait par «production propre»? Ce genre de production suppose l'application rigoureuse et constante du critère d'efficience écologique sans prétériter la capacité concurrentielle. Il s'agit d'un processus qui exige une grande capacité d'adaptation de la part des dirigeants de l'entreprise, car ils devront passer à un type de gestion nouveau, une gestion basée sur l'exploitation optimale de toutes les ressources. (L'entreprise dont les émissions équivalent à zéro n'est toutefois pas pour demain.) Les CPC travaillent dans une perspective à long terme et collaborent avec tous les acteurs concernés, les entreprises, l'Etat, les institutions financières, etc. Leur objectif est de mettre en place une coopération à long terme avec ces acteurs, pour s'adresser aux entreprises locales. Les $\mathrm{CPC}$ cherchent à coopérer avec des entreprises suisses pour mobiliser leurs connaissances et leurs capacités dans le domaine de l'environnement et les investir dans le développement.

Le Seco joue le rôle de mandant des CPC. En fait, il collabore avec des représentants locaux intéressés, avec d'autres donateurs ou encore avec l'Organisation des Nations Unies pour le développement industriel (ONUDI), afin de créer des centres nationaux ou régionaux. Chacun des CPC est en liaison avec un centre de référence en Suisse, qui lui sert de centre de compétences. Ces centres de compétence travaillent, pour leur part, avec des experts internationaux pour faire connaître les technologies environnementales les plus efficaces. L'objectif est de tisser un réseau international qui permettra aux différents CPC de communiquer entre eux, d'échanger leurs expériences et d'assurer leur formation réciproque, également dans le cadre des échanges Sud-Sud. La participation au réseau exige une attitude active de la part de tous: la principale tâche d'un CPC ne consiste pas à fournir ses propres prestations, mais à jouer le rôle d'un intermédiaire efficace, qui recherche, regroupe et transmet des prestations déjà disponibles sur les marchés local et international. Pour s'acquitter de cette tâche, les CPC font appel à des 
entreprises, à des consultants, à des universités et à des hautes écoles spécialisées suisses (LFEM/EMPA; FHBB - Haute école spécialisée des deux Bâles).

Un CPC compte quatre ou cinq collaborateurs, dont voici les principales activités : diffusion d'informations, développement de capacités, formation, réalisation de projets modèles pour sensibiliser le public cible et conseils destinés aux projets, notamment les conseils en matière de financement (comment et quand obtenir un crédit pour l'adoption d'une technologie nouvelle). Ces conseils servent aussi à rechercher les meilleurs moyens pour financer les adaptations technologiques. Les $\mathrm{CPC}$ cherchent à démontrer qu'une production plus efficace est aussi moins coûteuse et que l'efficience écologique contribue à accroître la rentabilité d'une entreprise. Des investissements plus importants ne s'imposent que dans un deuxième temps, notamment lors d'un agrandissement. Pour cette phase, on fait appel à d'autres instruments tels que des lignes de crédit spécifiques ou les fonds de capital-risque évoqués plus haut.

Les CPC collaborent avec des banques locales qu'ils tentent de motiver à offrir des lignes de crédit environnementales et à faire de l'efficience écologique un critère pour l'appréciation des demandes de crédit. La réforme du secteur bancaire en Bolivie va par exemple dans cette direction: les banques ne se fondent plus uniquement sur des garanties financières pour accorder un crédit, et s'attachent davantage à analyser la capacité de remboursement d'un projet d'investissement en tenant compte de son efficience, notamment aussi de son efficience écologique.

\section{Quelle est la contribution des CPC au développement?}

口 Au niveau de l'entreprise: En principe, ils augmentent la rentabilité des entreprises dans les pays en développement par les améliorations suivantes: accroissement de l'efficience, de la capacité concurrentielle, de la valeur intrinsèque de l'entreprise (lorsqu'elle veille par exemple à assainir ses sites contaminés dans le respect de l'environnement). En effet, les partenaires ou investisseurs potentiels accordent une grande place à la gestion des sites contaminés dans l'évaluation d'une entreprise.

口 Au niveau du pays: Les CPC contribuent au développement durable car ils poursuivent les objectifs suivants: préservation des ressources naturelles, exploitation optimale de l'énergie, amélioration des conditions de travail, réduction des émissions de substances nocives (d'où amélioration des conditions de vie de la population qui vit dans les zones industrielles), promotion de la formation et de l'apprentissage, création de capacités locales dans le domaine de l'environnement.

\section{Rôle des CPC pour l'industrie suisse}

Le relèvement ou l'égalisation des normes environnementales internationales simplifie toutes les relations commerciales. Les CPC fournissent des informations précieuses sur les besoins des pays en développement en matière environnementale, que le marché est à même de satisfaire. Nombre d'entreprises suisses, dont des PME, pourraient déployer des activités dans les pays en développement en collaborant avec les CPC.

La création et l'utilisation des nouveaux instruments présentés sont relativement récentes et il serait dès lors prématuré de vouloir procéder à une évaluation définitive. Il est néanmoins possible de tirer un premier bilan des données recueillies 
au cours des travaux préparatoires et de la réalisation des premiers projets, notamment aussi des premières observations faites sur le caractère novateur de ces nouveaux moyens de promotion.

\section{Comment fonctionne un CPC?}

Un patron s'adresse à un CPC pour lui demander, par exemple, comment adapter son entreprise aux normes environnementales internationales en vigueur. Le CPC examine sa situation et collabore avec ses centres de référence en Suisse (LFEM/EMPA, FHBB) pour élaborer une solution. Dans le cadre de cette collaboration, on fait appel à des experts et à des consultants, qui seront chargés de mener un examen approfondi de la situation avec l'entrepreneur intéressé. S'ils parviennent ensemble à mettre au point un projet modèle de qualité et à le mener jusqu'à l'adaptation des techniques de production au sein de l'entreprise, il sera possible d'adapter les méthodes de production de tout le secteur dans lequel travaille l'entreprise «modèle».

Un premier CPC a vu le jour en 1998 en Colombie. D'autres centres existent aujourd'hui au Salvador, au Costa Rica et au Guatemala, ainsi qu'au Vietnam et au Maroc (mis sur pied en collaboration avec l'ONUDI), au Brésil (sur une base bilatérale) et au Pérou (en collaboration avec les Etats-Unis). Des investigations sont actuellement en cours pour créer également des CPC en Chine et en Inde.

En Europe de 1'Est et dans la CEI, la situation est différente. Dès que ces pays ont commencé à s'ouvrir, l'industrie américaine a investi massivement dans leur secteur environnemental et les centres américains de prévention de la pollution ont accaparé le marché environnemental de ces pays. Comme leur nom l'indique, ces centres visent avant tout à éviter les atteintes à l'environnement, mais le traitement des déchets et les industries très polluantes demeurent un problème majeur dans les pays de l'Est. Le Seco examine actuellement la possibilité de mettre en ouvre l'instrument que sont les CPC dans ces pays.

\section{DÉBAT}

Nous avons demandé aux participantes et aux participants de nous fournir, avant la table ronde, une brève prise de position écrite sur les deux thèmes ci-après. Ces questions ont également servi à structurer le débat.

\section{A. INTÉRÊT DE L'ÉCONOMIE SUISSE}

- Quel intérêt l'économie suisse porte-t-elle aux nouveaux instruments de promotion et comment réagissent les PME?

- Quels sont les critères utilisés pour sélectionner les projets qui seront cofinancés dans le cadre de la coopération au développement et où se situe la différence avec le marché libre?

- Quelle est la politique d'information du Seco sur l'application des instruments examinés par la table ronde? Le Seco publie-t-il des listes de projets (investisseurs, partenaires, description de projets, etc.)?

\section{B. EFFETS SUR LE DÉVELOPPEMENT}

- Quelle est la répartition géographique des projets dans les pays bénéficiaires? 
- Comment mesure-t-on l'impact sur le développement?

- Quels sont les contacts entre les investisseurs, les responsables des instruments en Suisse, ainsi que les autorités et les groupements dans le pays en développement concerné?

- Quelle coordination est assurée entre ces instruments bilatéraux et les instruments multilatéraux de promotion des investissements (Société financière internationale [SFI] et Agence multilatérale de garantie des investissements [AMGI] de la Banque mondiale)?

\section{A. INTÉRÊT DE L'ÉCONOMIE SUISSE}

Jusqu'ici, l'économie suisse n'a pas manifesté un grand intérêt pour les nouveaux instruments du Seco destinés à promouvoir le secteur privé, les investissements et le transfert de technologies environnementales vers les pays en développement et en transition. Pourquoi cette réserve?

Thomas Bürki (consultant $C P C$ ): Les raisons de cette réserve ne sautent pas aux yeux. Cette attitude est peut-être due au fait que les PME suisses ne sont traditionnellement pas très orientées vers l'étranger et, lorsqu'elles le sont, les pays dans lesquels des CPC ont été créés ne figurent pas nécessairement sur la liste de leurs priorités. Une autre raison réside dans le manque de moyens mis en œuvre pour préparer et pour faire connaître ces instruments aux milieux intéressés parmi les PME. Pour expliquer leur attitude, les PME arguent aussi qu'elles font partie d'associations chargées de tâter le terrain, de réunir des informations et de leur préparer l'accès au marché.

Andreas Ragaz (SOFI): Les instruments de promotion, y compris la SOFI, sont encore trop récents pour être largement connus. J'ai d'ailleurs eu l'occasion de constater que d'aucuns ne connaissent pas non plus l'OSEC (Office suisse d'expansion commerciale), alors qu'il existe depuis quatre-vingts ans.

Fredy von Niederhäusern (ASICO, société ayant investi en Chine): La SOFI était une société toute jeune, pas connue du tout, et seul un heureux hasard m'a permis de la connaître: après de vaines démarches auprès de divers investisseurs potentiels (particuliers, banques, sociétés de participation) pour trouver les moyens de financer mon projet, j'ai lancé une recherche sur Internet avec pour mot clé «financement». C'est alors que j'ai trouvé la SOFI et que le premier contact a été établi. Nous avons ensuite développé le projet ensemble et il a pu être réalisé. L'accès aux informations concernant les instruments du Seco pose problème, mais il doit être difficile pour les administrateurs des fonds de diffuser les informations de manière à ce qu'elles atteignent le public visé sans déclencher un déferlement de demandes hors de propos. Dans le cadre des activités que j'exerce aujourd'hui pour la SOFI, en tant que membre de la commission d'experts chargée d'évaluer les demandes, je constate que la préparation, la formulation et la présentation des projets manquent souvent de professionnalisme. Il serait bon d'aider les PME à donner d'elles-mêmes une image plus professionnelle.

Susanne Grossmann (Seco): Il ne faut pas généraliser. Les fonds de partenariat bilatéraux - qui collaborent avec des entreprises suisses - se développent à notre entière satisfaction, comme le prouve la collaboration avec l'Inde et avec la Chine. La fréquence des investissements dans ces deux fonds est éga- 
lement relativement bonne. Tous leurs moyens financiers seront investis dans le courant de 2001 et le Seco se mettra bientôt en quête de nouveaux capitaux.

Les fonds de capital-risque locaux et régionaux ne s'adressent pas en premier lieu à l'économie suisse. Nous voulons bien entendu favoriser les partenariats entre entreprises suisses et locales, mais le secteur privé des pays en développement demeure au premier rang de nos priorités. En fait, il n'est pas facile d'inciter les entreprises suisses à conclure des partenariats, mais nous avons déjà enregistré quelques succès en Afrique du Sud.

Après deux ans d'existence, la SDSF n'est toujours pas au bout de sa traversée du désert. Les expériences faites à l'étranger (dans les pays scandinaves par exemple) avec des instruments similaires indiquent toutefois que ce démarrage très lent est classique. Nous devons donc nous armer de patience et nous attacher à susciter une plus grande confiance de l'économie, surtout des PME, à l'égard de la SDSF. N'oublions pas non plus que le capital-risque est une notion toute nouvelle pour les entreprises et pour le secteur financier en Suisse. A l'opposé, l'Angleterre et les Etats-Unis possèdent une longue tradition et une grande expérience en matière de financement du capital-risque.

Il est vrai toutefois que l'information pose problème et nous devons donc nous poser les questions suivantes: Comment communiquons-nous? Quelle est notre image auprès des entreprises? Jusqu'ici, nous avons surtout eu des contacts avec des associations et non pas directement avec les PME. Or les associations jouent le rôle de lobby des entreprises traditionnelles, d'une industrie habituée à exporter vers certains marchés. C'est pourquoi elles éprouvent peut-être une certaine aversion pour le risque. Par ailleurs, il ne faut pas que nos instruments dépassent les capacités des PME et qu'ils les attirent sur des marchés auxquels elles sont mal préparées. Peut-être devrons-nous tisser de nouveaux réseaux, faire preuve de plus d'initiative dans le domaine des nouvelles technologies et nous adresser directement à des entreprises dont les activités les poussent à innover et à prendre des risques. De telles entreprises existent et elles possèdent en général un potentiel de développement élevé.

Hans-Peter Egler (Seco): Le temps joue un rôle primordial avec ce genre d'instruments qui sont conçus pour le long terme. Or les premiers projets lancés ont à peine deux ans et font de plus intervenir des pays qui ne sont pas des partenaires traditionnels des exportateurs suisses (donc d'autant moins pour les PME).

Le secteur environnemental est très fragmenté en Suisse et surtout occupé par des PME. Il s'agit d'un secteur constitué de niches de production principalement axées sur le marché national et sur l'exportation vers les pays voisins (Union européenne). Le manque de protection de la propriété intellectuelle dans les pays en développement est un problème particulier qui ne fait qu'accroître la réserve des entreprises suisses, en particulier des PME. Il importe donc d'améliorer cette protection dans les pays en développement.

Nos mesures se concentrent sur les pays en développement et en transition. Les entreprises suisses sont un moyen pour réaliser nos objectifs et non pas la cible de nos activités. Nos instruments veulent stimuler l'innovation et ils exigent la participation d'investisseurs et d'entrepreneurs nouveaux, provenant de tous les secteurs et prêts à partager certains risques. Nous nous adressons 
donc à un nouveau groupe, à une nouvelle génération d'entrepreneurs. Et il faut du temps pour établir le dialogue. Dans ce processus, la recherche de canaux d'information appropriés joue en toute logique un rôle crucial.

Peter Bosshard (Déclaration de Berne): Je voudrais faire quelques remarques au sujet de la SFSD. Bien que je trouve judicieux de ne pas précipiter le financement de projets, force est de constater que le secteur privé suisse ne s'intéresse guère à cette offre. Même la mise sur pied de la SDSF a pris beaucoup plus de temps que prévu. A mon avis, ce retard n'est pas dû à la politique d'information du Seco, mais à une question plus fondamentale : celle qui concerne la raison d'être de cette institution. Pourquoi avoir créé la SDSF? En fait, il s'agissait pour le Seco d'imposer dans notre pays le principe qui prévaut au niveau international - surtout au sein des institutions de la Banque mondiale - et qui veut que le développement passe inévitablement par la promotion du privé. Pour aiguiller la coopération suisse au développement sur cette voie, le Seco avait besoin d'un instrument bien à lui. Par conséquent, nul n'a jamais dû prouver qu'il était nécessaire de créer une telle institution en Suisse. Sa création partait d'une intention louable mais se fondait aussi sur une motivation idéologique.

Bien entendu, la coopération au développement doit aussi pouvoir faire ses expériences et les nouvelles approches apportent toujours une foule d'enseignements. Ce qui me préoccupe toutefois, c'est le manque d'intérêt de l'économie pour ce genre d'instrument. A mon avis, lorsque l'on s'est aperçu, pendant la phase préparatoire, que l'économie privée n'était pas emballée, on lui a accordé trop de concessions, surtout pour ce qui est de la politique et des structures de la SDSF, pour que le projet ne capote pas. A titre d'exemple, je citerai la garantie contre les risques politiques, dont nul n'avait parlé à l'origine. Des concessions ont également été consenties en matière de transparence: contrairement aux promesses initiales, les directives en matière d'investissement ne sont pas publiées et demeurent vagues. Par ailleurs, le Seco n'est pas représenté au sein de la Commission d'investissement de la SDSF. A notre avis, nul ne veille donc à ce que les projets cofinancés par la SDSF soient compatibles avec la politique de développement, et les projets ne font pas l'objet d'une publication officielle. Nous craignons et regrettons que la SDSF souffre de ces lacunes et qu'elle ne soit plus à même de jouer le rôle qui lui était dévolu dans la politique de développement.

Andreas Ragaz: Ma remarque est également d'ordre idéologique et concerne la promotion des investissements et le développement. A l'aide des instruments de promotion étatiques, nous tentons de combler des niches et de colmater des brèches. Nous jouons ainsi un rôle que l'économie privée n'est pas prête à assumer, car elle juge les risques trop grands. De plus, nous ne voulons pas que ces instruments servent tout bonnement à subventionner des entreprises et qu'ils faussent les marchés, car nous ne voulons pas maintenir artificiellement à flot des entités incapables de subsister à long terme sur le marché. Pour appliquer cette approche, il faut définir clairement les activités. Or cette délimitation crée souvent des lacunes dans le financement d'un projet; lacunes que l'Etat n'est pas autorisé à combler, et que les banques ou investisseurs privés ne sont, pour leur part, pas prêts à financer. Cette offre lacunaire est peutêtre aussi l'une des causes du manque d'intérêt de la part des PME. 
Fredy von Niederhäusern: Il ne faut pas confondre le fait que les instruments qui nous intéressent sont mal connus avec le manque d'intérêt du secteur privé. En fait, les PME ont un grand besoin de mesures de soutien et d'incitation pour mener des projets, notamment lorsqu'il s'agit de financer le capital-risque destiné à des petits projets. Le mécanisme des sociétés de participation privées et bancaires n'est toutefois guère attrayant pour les PME. Cependant, dans le cas des sociétés de participations soutenues par le Seco, les conditions de rachat sont clairement établies d'entrée de jeu et les taux d'intérêts sur le capital sont abordables.

Il se peut toutefois qu'en créant ses nouveaux instruments financiers, le Seco ait pris trop d'avance sur les entreprises, qu'il mène une politique novatrice que tout le monde ne comprend pas encore. Dans ce cas, il faut améliorer l'information.

Nadine Keim (Communauté de travail des auvres d'entraide): Dans les pays en développement, l'intérêt que suscitent la promotion et le soutien de l'économie privée est au moins aussi grand que les besoins recensés dans ce domaine. Il importe donc de faire connaître ces besoins aux acteurs de l'économie suisse et de les inciter à s'engager dans les pays en développement. Je m'interroge toutefois sur un point: les instruments dont nous sommes en train de parler ont été créés au milieu des années 90, soit avant l'entrée en vigueur de la nouvelle loi fédérale sur la promotion des exportations. Comment les instruments de développement du Seco s'insèrent-ils à présent dans les mesures de promotion des exportations prévues par la loi? Quels sont les avantages de ces instruments de développement face aux mesures conçues uniquement pour promouvoir les exportations, d'autant que les critères de développement des instruments du Seco sont formulés de manière très floue?

Susanne Grossmann: La promotion des exportations n'a rien à voir avec les instruments dont nous parlons ici.

Nadine Keim: Les documents accessibles au public qui présentent ces instruments reviennent sans cesse sur la promotion des exportations et de l'économie suisse.

Susanne Grossmann: Les nouveaux instruments de développement du Seco ont pour objectif de promouvoir le secteur privé dans les pays en développement et en transition, ainsi que de favoriser les investissements tournés vers ces pays. Leur objectif n'est pas de promouvoir les exportations suisses. Il n'est du reste pas toujours facile de faire comprendre à nos partenaires économiques traditionnels qu'il ne s'agit justement pas de promotion des exportations, mais que nous leur demandons en fait d'assumer des risques et d'investir dans les pays en développement et en transition.

Peter Bosshard a également mentionné les besoins du secteur privé. Il convient ici de distinguer les clients de la SDSF, parmi lesquels notamment les PME suisses et les investisseurs. Les investisseurs ont la possibilité - et je n'invente rien - d'obtenir de hauts rendements moyennant peu de risques sur les marchés «traditionnels» de l'investissement. Les investissements que nous proposons dans les pays en développement n'ont donc aucun attrait à leurs yeux. Mais même ce constat ne suffit pas pour dénier toute raison d'être à la SDSF. La SDSF n'a en effet pas pour but d'offrir aux investisseurs des 
fonds garantissant un rendement maximum, mais bien plus de leur faire partager notre responsabilité à l'égard des pays en développement.

Pour ce qui est de la critique concernant le manque de transparence, je n'ai encore jamais entendu dire que les directives d'investissement d'un fonds de capital-risque soient tenues secrètes. Au contraire, ces directives doivent être connues, car elles servent de base à l'évaluation d'une entreprise, donc pour savoir si un projet mérite d'être soutenu par le fonds. Pour ce qui est du rôle du Seco au sein des instances dirigeantes, il faut bien comprendre que la SDSF est une entreprise privée avec une participation de la Confédération. Le Seco est représenté au sein du conseil d'administration de la SFSD qui a adopté les lignes directrices en matière d'investissement. Pour pouvoir s'acquitter de sa tâche, la direction privée de la SDSF doit toutefois jouir d'une grande liberté en matière d'attribution des moyens et de sélection des projets, mais il est bien entendu que cette liberté ne peut s'exercer que dans le cadre délimité par les lignes directrices en matière d'investissement. Le message du Conseil fédéral prévoit enfin que la rentabilité et le respect des critères de développement de la SDSF et du fonds de capital-risque devront faire l'objet d'une évaluation au terme d'une période de cinq ans.

Pour ce qui est du reproche concernant l'insuffisance de l'offre, je dois dire que le Seco est tenu de respecter les exigences d'ordre politique, c'est-à-dire que nous ne pouvons pas fausser la concurrence, nos actions doivent se soumettre aux lois du marché. Cette position nous vaut des critiques des deux côtés : pour les uns, nous nous concentrons sur des domaines trop étroits, alors que les autres jugent que nos instruments sont trop nombreux et nos activités trop dispersées.

Nous avons tenu compte de la critique concernant les lacunes de notre offre et sommes déjà en discussion avec la SOFI à ce sujet.

Hans-Peter Egler: Les instruments de développement du Seco suscitent des attentes contradictoires. Il y a d'une part, les attentes de l'économie privée, qui suivent les lois de l'économie de marché; et d'autre part, les attentes de groupements qui ne comprennent pas très bien cette orientation. Notre approche est la suivante: le secteur privé doit être le moteur, et il doit en principe assumer le risque lié à son investissement et manifester un intérêt durable pour ce genre d'opération. En réalité, de tels investissements font l'objet d'examens détaillés. La décision n'intervient donc qu'au terme d'un processus relativement long. Ce processus est toutefois important car il permet de donner des conseils à l'auteur du projet, de retravailler le projet lorsque c'est nécessaire, ainsi que d'examiner et d'améliorer les éléments relevant du développement et de la politique environnementale.

Pour ce qui est des CPC, les investigations sont approfondies. Le public cible d'un CPC est clairement défini : il s'agit des PME du pays d'implantation. Le centre leur offre des prestations complètes (conseils et suivi d'un projet). Un CPC est ainsi à même de conseiller un entrepreneur sur la manière d'optimiser l'organisation du travail au sein de son entreprise ou d'une entreprise future. Et cette efficacité fondamentale inclut bien entendu aussi l'efficience écologique. Nous offrons donc aux entreprises la possibilité d'accroître leur efficience et leur rentabilité. Le fait qu'elles garantissent ainsi une meilleure 
qualité environnementale n'est qu'un effet secondaire important et appréciable. N'oublions pas que les appels à la morale n'ont jamais eu aucun effet sur les entrepreneurs.

Dans le cadre des CPC, nous avons défini des indicateurs de politique du développement à l'instar de ce qui se fait pour un projet pilote. Les projets sont évalués et, le cas échéant, réorientés tous les six mois sur la base de ces critères.

Peter Bosshard: Le Seco part du principe que quiconque veut collaborer avec le secteur privé doit aussi accepter les lois de ce secteur. Les instruments dont nous parlons ici nous amènent toutefois dans un secteur mixte et nous pouvons donc également exiger que les entreprises privées respectent certaines règles des pouvoirs publics lorsqu'elles utilisent les fonds publics. Ces règles comprennent des contrôles, l'obligation de rendre compte de ses activités et la transparence. J'ai été heureux d'entendre que les projets et les directives d'investissement font l'objet d'une information complète. La SFI fait d'ailleurs de même.

Pour ce qui est de veiller au respect de la politique de développement, l'OCDE regrette justement, dans son rapport consacré à la Suisse, que le Seco ne soit pas du tout représenté dans la commission d'investissement de la SDSF. La Déclaration de Berne et les œuvres d'entraide avaient déjà formulé cette critique auparavant. Nous pensons donc que nous devons améliorer le contrôle du respect des critères de développement dans le cas de la SDSF.

Thomas Bürki: Il est encore trop tôt pour tirer des conclusions définitives sur l'engagement de l'économie privée et sur l'efficacité des instruments de promotion. N'oublions pas que nous n'en sommes qu'au stade où l'on prépare le terrain à des investissements efficaces en faveur du développement. Ceux-ci permettront ensuite aux CPC d'améliorer l'efficience des industries de pointe dans les secteurs environnemental et énergétique et d'accroître l'efficience globale des entreprises des pays en développement. Il s'agit donc de commencer par susciter l'intérêt des entreprises et d'organiser le transfert de connaissances et de technologies vers ces pays. D'ici deux à trois ans, nous aurons une expérience plus grande et les entreprises s'intéresseront plus à nous. En Suisse, nous nous adressons en effet avant tout aux PME jeunes, modernes et souples, qui sont prêtes à investir à l'étranger, mais au-delà l'Union européenne. C'est juste une question de temps, qui ne remet pas en cause le bien-fondé des instruments utilisés.

\section{B. EFFETS SUR LE DÉVELOPPEMENT}

Les instruments du Seco que nous évoquons ici partent de l'idée que la promotion du secteur privé contribue grandement au développement. Pour s'acquitter de son mandat, qui est de lutter contre la pauvreté, la DDC compte aussi beaucoup sur le rôle de l'économie privée et sur sa contribution au développement socio-économique. Comment considère-t-elle les instruments du Seco dans ce domaine? Quels sont les recoupements? Qu'en est-il des indicateurs permettant de mesurer l'impact sur le développement?

Urs Heierli $(D D C)$ : La DDC a pour mandat de lutter contre la pauvreté et le secteur privé joue dans ce cadre un rôle important. A notre avis, il est essentiel que ce soit le secteur privé et non pas le secteur public qui crée et qui 
propose des emplois. En effet, les petites et les micro-entreprises sont un élément clé de l'emploi. C'est pourquoi la DDC souhaite créer des synergies entre les instruments du Seco et les siens. J'ai toutefois l'impression que l'application des instruments variera selon les pays, puisque nous travaillons principalement dans des pays très pauvres.

Nous élaborons en ce moment un concept de promotion du secteur privé, mais nous avons une conception très large de ce secteur dans les pays en développement : pour nous, un petit paysan fait partie du secteur privé, ce qui n'est pas l'avis du Seco. Le défi que nous tentons de relever depuis quelques années se résume à la question suivante: Comment atteindre les groupes les plus pauvres, les plus faibles et les plus désavantagés de ce secteur privé?

Nous constatons en outre que les instruments classiques ont presque toujours échoué dans ce domaine. Susanne Grossmann a d'ailleurs déjà donné la raison de ces échecs: le coût des transactions. Nous ne pouvons appliquer ici les normes habituelles et les critères conçus pour le secteur formel. Nous devons plutôt exploiter ce que nous a appris la «révolution» de la microfinance, qui doit justement son succès au fait d'avoir abaissé les coûts de transaction.

Reste à savoir comment atteindre les gens qui n'ont accès ni au capital, ni au savoir, ni à la technologie. Cette remarque vaut surtout pour les PME, pour qui les difficultés ici et dans le Tiers Monde sont d'ailleurs souvent similaires. L'innovation est de mise. La mise en œuvre des instruments du Seco nous fournit des expériences précieuses et nous recueillons aussi des informations sur le leasing. Celui-ci possède en effet un potentiel phénoménal lorsqu'il s'agit de mettre du capital et des machines à disposition des petites entreprises. Une étude consacrée à un projet de leasing au Pakistan a examiné les avantages et les inconvénients de cet instrument, et cette étude a été présentée au Comité des donateurs.

Pour promouvoir les PME, nous tablons sur la promotion de services pour le développement d'entreprises (Business Development Services, BDS). Autrement dit, nous soutenons des sociétés privées locales qui vendent des services aux petites entreprises. Nous voulons créer des débouchés pour leurs activités, sans fausser les marchés mais en offrant de nouveaux services en vue de promouvoir et d'améliorer les PME locales. Nous n'en sommes toutefois qu'aux essais et la question du subventionnement nous préoccupe beaucoup. Nous n'avons toutefois pas de recette toute prête.

Susanne Grossmann: La DDC et le Seco poursuivent les mêmes objectifs. Le secteur privé a d'énormes besoins, notamment de moyens pour financer des projets et des programmes à différents niveaux. Dans ce cadre, les activités de la DDC et du Seco sont complémentaires. Et en échangeant nos expériences sur le microfinancement (DDC) et sur la promotion de plus grandes entreprises (Seco), nous pourrions créer des synergies. Nous aussi, nous avons constaté que le leasing donne de bons résultats, notamment dans des zones urbaines d'Afrique. Le Seco s'est doté de directives sur l'évaluation et a nommé un responsable dans ce domaine, qui pourrait coordonner nos échanges d'information. 
Susanne Grossmann: Le Seco reprend les indicateurs que la SFI et la Banque mondiale utilisent pour évaluer des instruments similaires. La SFI a en effet procédé à une évaluation exhaustive de ses intermédiaires financiers en Afrique et nous nous référons à cette évaluation pour élaborer des indicateurs pour nos propres fonds. Nous voulons pouvoir mesurer aussi bien la rentabilité que l'impact sur le développement. Pour ce dernier, la création d'emplois est un indicateur de premier ordre.

Nadine Keim: Nous serions heureux de participer à l'élaboration de critères du développement et d'indicateurs de l'impact sur le développement, de même que nous sommes prêts à suivre activement les travaux de la SDSF. Pour ce qui est des critères du développement, je tiens à relever que les œuvres d'entraide n'ont, depuis le message de 1995, reçu aucune information directe sur les instruments du Seco. Les informations sur Internet ne contiennent aucune indication précise sur les critères en vigueur. Applique-t-on les mêmes critères à tous les instruments ou bien leur définition varie-t-elle selon l'instrument considéré ? Ces instruments sont-ils en adéquation avec les instruments multilatéraux ? Bref, nous manquons cruellement d'informations sur ce sujet.

Peter Bosshard: Nous sommes intervenus lors des travaux préparatoires pour exprimer les souhaits des organismes de développement et des auvres d'entraide en ce qui concerne les différents critères à respecter. Nous avons par exemple demandé que les instruments se concentrent plus particulièrement sur les populations et les pays les plus pauvres. D'autres critères comprennent des conditions de travail exemplaires et la promotion de produits qui favorisent une économie durable. Nous avons aussi suggéré que la gestion ne soit pas jugée uniquement sur la base des rendements financiers, mais aussi selon sa politique de développement. Lorsque ce dernier critère disparaît, la direction de l'entreprise ne vise plus que le rendement économique et renonce à investir dans le social.

Fredy von Niederhäusern: A mon avis, la création de bons emplois est un indicateur du développement important. En tant qu'investisseurs étrangers dans des pays où les conditions de travail sont pitoyables, nous devons jouer le rôle de pionniers en offrant de bonnes prestations aux employés: salaire, assurances sociales, propreté et sécurité au travail, allocation d'entretien, etc. Dans notre entreprise en Chine, les repas servis à midi à la cantine sont gratuits, les habits de travail sont mis à disposition gratuitement et les salaires sont supérieurs à la moyenne. Nous respectons aussi les prescriptions environnementales et gérons nous-mêmes nos déchets. Avec l'aide de la SOFI, nous avons ainsi créé cinquante postes de travail exemplaires en Chine.

Andreas Ragaz: Nos critères de sélection des projets comprennent la création d'emplois, le maintien de la plus-value dans le pays et la rentabilité économique. Le comité d'experts chargé d'examiner les demandes réunit aussi, outre des représentants des entreprises et du Seco, un représentant de l'organisation de développement Swisscontact, ainsi qu'un expert de l'environnement et un membre du centre spécialisé dans le transfert de savoir-faire à l'EPF de Zurich. Ces experts évaluent la rentabilité et l'impact sur le développement des projets qui leur sont soumis. Les entreprises que nous 
appuyons ensuite sont tenues de nous rendre compte de leurs activités (rapport) et de nous remettre un rapport final lorsque le crédit arrive à échéance. Après cette étape, nous ne suivons plus le projet et ne procédons donc pas à une évaluation ultérieure (ex-post). Nous n'avons toutefois pas développé d'autres critères, aussi faciles à appliquer que la création d'emplois.

Hans-Peter Egler: Mesurer l'impact des projets sur le développement est essentiel, mais extrêmement difficile. Le Seco a mis au point un système de monitoring pour suivre les activités des CPC et pour mesurer leur impact à différents niveaux. Les projets soutenus peuvent en effet engendrer des problèmes auxquels nul n'aurait pensé au départ. Il est par exemple possible que la création - soutenue par l'un de nos instruments - d'une entreprise qui offre 40 emplois stables et rentables réduise par ailleurs à néant la capacité concurrentielle de 100 micro-entreprises du même secteur. Avons-nous alors accéléré une évolution qui serait intervenue de toute manière? Avons-nous créé des emplois stables pour les 40 salariés de la nouvelle entreprise et, parallèlement, incité plusieurs centaines de micro-entrepreneurs - désormais non compétitifs - à innover? Ou avons-nous condamné ces derniers à la misère ? Quel est le bilan d'une telle opération? Un bienfait social? Dans quelles limites mesure-t-on l'impact sur le développement? La DDC possède une grande expérience dans le domaine des tanneries, par exemple, qui ont amélioré les conditions de logement, et réduit les émissions d'odeurs et de mercure. Il serait très intéressant, tant pour le Seco que pour la DDC, d'établir un dialogue sur la manière de mesurer l'impact sur le développement et pour faire connâtre quelques cas exemplaires (best practices).

Susanne Grossmann: Au Seco, nous serons heureux de recevoir les données que la DDC et les ONG voudront bien nous fournir sur la manière de mesurer l'impact sur le développement.

Andreas Ragaz: L'évaluation des projets devrait porter sur une période plus longue.

Susanne Grossmann: Du point de vue du développement durable, je suis d'accord. Les projets devraient être suivis sur une période plus longue.

Hans-Peter Egler: Nous nous efforçons en ce moment de compiler les diverses normes - gestion, environnement, secteur social - pour en faire un ensemble de normes de durabilité.

Urs Heierli : Il est relativement facile de mesurer l'impact sur le développement au niveau micro-économique. Puisqu'elle souhaite toutefois aussi exercer une grande influence sur la promotion du secteur privé, la DDC est également très active au niveau méso où elle tente de toucher le maximum de petits entrepreneurs, de même qu'au niveau macro où elle tente de créer des conditions politiques plus favorables aux PME. Plus le niveau sur lequel on travaille est vaste, plus il est difficile de mesurer l'impact, mais l'impact est d'autant plus grand. Nous devons savoir qu'il n'est pas possible de tout contrôler et de tout mesurer. Compter les emplois ne suffit pas pour connaître l'impact sur le développement.

Nadine Keim: La création d'emplois ne suffit pas à elle seule, mais elle n'en reste pas moins un élément important de la promotion du secteur privé. Il ne faut certes pas compliquer les choses, mais il convient tout de même de res- 
pecter certains critères, par exemple le coût d'un poste de travail par unité de capital investi.

Pour terminer, je voudrais dire que les œuvres d'entraide ne sont en principe pas opposées aux instruments de la politique financière et commerciale du Seco. Au contraire, nous saluons leur création et leur caractère novateur. Nous tenons toutefois à souligner que le nouveau crédit de programme du Seco sera débattu d'ici un à deux ans déjà et qu'il serait bon que certains instruments du $5^{\text {e }}$ crédit évoqués ici fassent auparavant l'objet de bilans sérieux et d'évaluations détaillées. Les rapports de monitoring ne suffisent pas. Les remarques de l'OCDE concernant la coopération suisse au développement relèvent d'ailleurs la grande diversité des approches et le trop grand nombre de pays bénéficiaires. L'OCDE reproche par ailleurs au Seco de ne pas procéder systématiquement à des évaluations. Cette critique doit aussi être prise au sérieux. Enfin, il reste à savoir dans quelle mesure les instruments de promotion du Seco stimulent effectivement le développement et dans quelle mesure ils servent à promouvoir les exportations suisses. A mon avis, cette question reste ouverte.

Hans-Peter Egler: Nos instruments de développement ne servent pas à financer certaines exportations, mais à promouvoir la coopération technique et le transfert de savoir-faire. Il faut considérer cette palette d'instruments comme un tout. On se rend alors compte de l'importance accordée à la collaboration avec l'économie - qu'il s'agisse d'entreprises isolées ou d'associations - avec la DDC et avec les œuvres d'entraide, dans la conception et dans la mise en œuvre des mesures. Le Seco collabore par ailleurs avec les principaux organismes internationaux de financement et avec les institutions de l'ONU; il coordonne aussi ses activités avec d'autres donateurs bilatéraux et réalise des projets avec eux. A l'avenir, le travail en réseau sera le maître mot de nos activités, c'est pourquoi le Seco s'efforce de mettre en place un processus commun qui fera intervenir le maximum de partenaires pour promouvoir le développement.

Gérard Perroulaz: Nous abordons ici des éléments importants liés aux critères environnementaux et sociaux des instruments de promotion. Or ces éléments ne figurent absolument pas dans les documents du Seco. Une question reste toutefois en suspens: Il s'agit de savoir si ces nouveaux instruments correspondent encore aux critères de l'aide publique au développement tels qu'ils sont définis par l'OCDE, ou si leur volet consacré à la promotion des investissements - SDSF et SOFI - ne les classe pas dans une autre catégorie de l'aide publique. La limite n'est pas claire.

Susanne Grossmann: Les critères de l'OCDE sont trop réducteurs, car ils ne distinguent que deux catégories d'aide: les cadeaux (ou dons) et les crédits.

Gérard Perroulaz: Dans son classement, l'OCDE tient toutefois aussi compte des objectifs des instruments. Ceux-ci profitent-ils avant tout aux pays en développement ou soutiennent-ils autant l'économie suisse?

Pour ce qui est des critères du développement, un rapide coup d'œil à la liste des projets financés me donne à penser qu'ils ne sont pas tous soumis au même traitement. L'appui accordé à des boulangeries qui fabriquent des baguettes parisiennes en Afrique du Sud est-il vraiment prioritaire pour le développement? Et qu'en est-il de la promotion de la culture hors sol pour exporter des fleurs vers le marché suisse? 
Aujourd'hui, nous n'avons examiné en détail que trois des instruments du Seco et leur coordination pose déjà des problèmes. Or le Seco dispose d'une vaste palette d'instruments et je pense donc que l'OCDE a raison de dire que ces instruments sont trop nombreux.

Peter Bosshard: Il importe de promouvoir la durabilité sociale et écologique à tous les niveaux (micro, méso et macro). Au niveau de l'entreprise, il est relativement simple de connaître l'impact sur le développement. Pour assurer la cohérence de notre politique économique extérieure, il importe toutefois de tenir davantage compte des aspects sociaux et écologique également au niveau macro. Les mesures d'ajustement structurel, les aides à la balance des paiements et les accords de protection des investissements devraient ainsi tenir compte des droits syndicaux et des prescriptions environnementales.

Thomas Bürki: Il est clair que les CPC se concentrent sur les dimensions écologique et économique du développement. Les aspects sociaux ne sont à l'évidence pas au centre de leurs préoccupations, bien que les conditions de travail revêtent parfois une grande importance. S'il existait un instrument qui permettrait de prendre en compte au même titre toutes les dimensions du développement et qui serait en même temps opérationnel, on l'aurait déjà inventé. Puisque nous devons travailler avec plusieurs instruments, il est logique que chacun ait ses priorités. Les CPC semblent promis à un beau succès, mais n'en sont qu'à leurs débuts. Puisque nous plaidons en faveur du travail sur le long terme, notre jugement ne peut pas être définitif après un à deux ans de fonctionnement.

Fredy von Niederhäusern: Grâce à l'appui de la SOFI, nous avons pu consolider nos emplois en Suisse et changer de statut: de revendeur, nous sommes devenus producteur et avons amélioré nos perspectives d'avenir. De plus, nous avons créé des emplois rentables et stables dans un pays relativement pauvre (la Chine). Je pense donc que l'instrument mis en œuvre est valable et suis persuadé que l'économie privée a besoin de ce genre d'instrument. Par ailleurs, je trouve que les entreprises qui bénéficient d'un soutien pour investir dans les pays en développement ne peuvent qu'adopter une politique exemplaire dans tous les domaines (environnement, secteur social et économique), si elles veulent pouvoir commercialiser leurs produits en Suisse. C'est le prix à payer pour bénéficier du soutien des pouvoirs publics.

Andreas Ragaz: Dans l'ensemble, les instruments du Seco vont dans la bonne direction, mais il reste encore beaucoup à faire en matière d'information, de sensibilisation, de coordination et d'évaluation. Je me demande s'il ne serait pas temps pour la Suisse de créer une banque pour le développement, à l'image de la «Kreditanstalt für Wiederaufbau» allemande. Cette banque s'occuperait des différents fonds et instruments. Les activités seraient ainsi moins dispersées, moins sectorielles et moins ponctuelles, et la banque contribuerait à optimiser les forces en présence. Une telle banque pourrait proposer un portefeuille complet aux investisseurs et aux entreprises privées.

Urs Heierli: Je pense que nous devrions suivre de près les effets successifs de nos mesures, et aussi tenir compte des activités qui précèdent et qui suivent. Un tel suivi va dans le sens de la durabilité et éviterait à nos projets - dont la valeur éthique est reconnue - d'apparaître comme des îlots artificiels sans aucun lien avec la réalité qui les entoure. 
Susanne Grossmann: Je trouve que des débats aussi ouverts que celui-ci revêtent une importance d'autant plus grande que nos instruments sont sujets à controverse. Nous devrions aussi échanger en toute franchise nos avis sur un autre domaine essentiel : l'évaluation et l'exploitation des expériences. Le développement a de multiples facettes et la mise en œuvre des instruments examinés ici est pleine d'inconnus tant pour le Seco que pour la DDC. Nous ne pourrons peut-être pas prouver que la promotion du secteur privé exerce une influence directe sur la lutte contre la pauvreté, mais elle y contribue certainement.

En matière de coopération, nous devons certes nous fixer des objectifs ambitieux, mais sans devenir puristes. Nous devons agir de manière cohérente et logique, c'est-à-dire aussi ouvrir nos marchés aux entreprises des pays en développement. 


\subsection{PRISES DE POSITION}

\section{RÉFLEXIONS SUR LES EXPÉRIENCES DES NOUVEAUX INSTRUMENTS DU SECO}

\section{SUSANNE GROSSMANN}

\section{$\square$ Quel intérêt l'économie suisse porte-t-elle aux nouveaux}

instruments de promotion du Seco et comment réagissent les PME?

L'intérêt que suscitent les nouveaux instruments du Seco destinés à promouvoir le secteur privé et les investissements varie selon l'instrument. Les prestations d'entremise et d'appariement d'entreprises de la Swiss Organization for Facilitating Investments (SOFI) et les crédits du fonds d'études administré par la SOFI font ainsi l'objet d'une demande stable. De même, les deux fonds de partenariat existants (Switcher en Inde et Sinop Twist Partenariaux Fundus, SSPF, en Chine) sont assez actifs. Compte tenu du cadre dans lequel ils opèrent, ces deux fonds présentent une bonne fréquence d'investissement et des relations satisfaisantes. Quant à la Société financière suisse pour le développement (SFSD), elle doit encore s'ouvrir de nouveaux débouchés et tisser des réseaux sur place. Elle pourra ainsi améliorer ses relations d'affaires. Il nous paraît prématuré de tirer des conclusions définitives quant au fonctionnement de la SFSD, mais nous allons suivre de près l'évolution de cet instrument et, le cas échéant, lui apporter des modifications ou des améliorations. Pour ce qui est des fonds locaux de capitalrisque, ils accordent à l'évidence la priorité à la promotion du secteur privé local et régional. Des investisseurs suisses - parmi lesquels même des PME - ont néanmoins pu profiter de leurs services (p. ex. en Afrique du Sud et en Afrique du Nord).

Voici quelques indications dont il faut tenir compte lors de l'évaluation des instruments mentionnés :

- Les instruments de promotion du secteur privé ne réservent pas la même place aux entreprises suisses. Les fonds locaux de capital-risque n'accordent par exemple aucune préférence aux investisseurs suisses.

- Le fonctionnement de ces nouveaux instruments se fonde uniquement sur des critères commerciaux. Par conséquent, la sélection des projets est impitoyable (seuls les meilleurs sont sélectionnés) et ce processus de sélection suscite à n'en point douter des réactions critiques.

- Nous mettons les instruments de promotion (ou facilités) à disposition des investisseurs, mais l'usage qu'en font les entreprises suisses dépend (bien plus qu'auparavant) de leur propre volonté et de leur propre engagement.

- Les nouveaux instruments reposent avant tout sur le principe du partage du risque et du profit.

- L’intérêt des investisseurs suisses varie énormément selon la région.

Quels sont les critères utilisés pour sélectionner les projets qui seront cofinancés dans le cadre de la coopération au développement et où se situe la différence avec le marché libre?

Les critères de base sont définis dans la législation sur la coopération au développement, ainsi que dans les crédits de programme 5 (Sud) et 3 (Est), c'est- 
à-dire dans les messages qui les présentent. Dans le cas des nouveaux instruments de promotion du secteur privé, la viabilité financière et commerciale joue toutefois un rôle important pour garantir la durabilité de projets d'investissement privés. Relevons en outre que nous n'acceptons de mettre nos instruments en œuvre que lorsqu'un certain cadre économique et politique est garanti (stabilité macro-économique, existence de potentiels économiques suffisants).

La différence par rapport au marché libre réside par exemple dans la subsidiarité, c'est-à-dire que les nouveaux instruments du Seco doivent rester subsidiaires au marché. Des subventions ciblées (aides non remboursables, prêts à des conditions favorables) se limitent aux secteurs où elles sont en mesure de mobiliser un grand volume de ressources privées (le Seco sert de catalyseur), par exemple pendant la phase d'investissement primaire (partage du risque avec le secteur primaire), pour renforcer l'équipe de gestion locale et favoriser une bonne gestion de l'entreprise, ainsi que pour améliorer le climat d'investissement global. Le Seco joue également le rôle d'investisseur auprès d'intermédiaires financiers en faveur de régions, de catégories d'entreprises ou de secteurs, qui n'ont pas (encore) accès au capital privé; cet accès étant souvent interdit par une perception faussée du rapport entre risque et rendement.

Contrairement aux conditions prévues par les instruments traditionnels que sont les crédits mixtes et les garanties de crédit, les entreprises et les investisseurs privés suisses ne jouissent pas d'un traitement préférentiel (sauf dans le cas de la SOFI, du fonds d'études, des fonds de partenariat et de la SFSD).

Quelle est la politique d'information du Seco

sur l'application des instruments de promotion? Le Seco publie-t-il

des listes de projets (investisseurs, partenaires, description de projets, etc.)?

Nous utilisons des moyens d'informations différents selon les instruments mis en œuvre:

- Brochures en allemand/anglais/français sur nos instruments.

- Site Internet détaillé.

- Présentations à l'extérieur (p. ex. à l'occasion de la Conférence annuelle de la coopération suisse au développement, EMA, économie suisse, etc.).

- Rapports au Parlement.

- Messages, bureaux de coordination de la DDC.

Trois des quatre secteurs opérationnels dans le domaine de prestations Développement et transition tiennent à jour des listes des projets en voie de réalisation. Il est prévu d'établir de telles listes pour tous les secteurs et de les publier sur notre site Internet. Ces listes contiennent une brève description de chaque projet, ainsi que les indications suivantes: pays, secteur, montant, durée et partenaire(s).

\section{$\square$ Quelle est la répartition} géographique des projets dans les pays bénéficiaires?

- En principe, nous ne répartissons pas nos projets du point de vue géographique, mais nous nous efforçons plutôt de les concentrer sur une région donnée.

- Les pays bénéficiaires sont choisis sur la base de critères non seulement politiques (nous mettons par exemple l'accent sur l'Asie centrale, car les pays de 
cette région appartiennent à notre groupe de vote au sein de la Banque mondiale et du FMI), mais aussi en fonction de l'impact potentiel de nos instruments. Cet impact pouvant être obtenu par la mise en œuvre successive (en Europe centrale, par exemple, l'aide financière a été remplacée par une application ciblée des instruments de promotion du commerce et des investissements) ou parallèle de ces instruments (exemple: Mozambique 2001).

- Nous parvenons ainsi à concentrer les instruments sur certains pays ou régions: CEI et Europe de l'Est, Balkans, Inde, Chine, Afrique du Nord, sud de l'Afrique (Afrique du Sud, Mozambique dès 2001), Brésil et Amérique centrale.

- Il reste des régions dans lesquelles nous voulons encore développer notre présence: Afrique occidentale (surtout aussi les pays francophones), Andes, ASEAN, républiques d'Asie centrale/Caucase (groupe de vote au sein des institutions de Bretton Woods).

- Les nouveaux instruments mettent l'accent sur les zones urbaines.

\section{$\square$ Comment mesure-t-on l'impact sur le développement?}

Toutes nos activités sont en principe soumises à des évaluations régulières, aussi bien internes qu'externes. Ces évaluations sont coordonnées par un responsable, et elles sont planifiées et réalisées en accord avec les divers secteurs. Les collaborateurs ont reçu des directives (manuel) pour évaluer les résultats de la coopération économique avec les pays en développement et en transition. Ce manuel est régulièrement adapté aux développements les plus récents, et fait partie intégrante de notre système de gestion de la qualité, lui-même régi par la norme ISO 9001.

Le manuel se fonde sur une approche dite des «cycles de gestion des projets» et son application garantit l'établissement de liens entre la planification, le suivi en cours de réalisation (monitoring), ainsi que les contrôles en fin de projet et a posteriori. Selon les besoins, les contrôles de résultats interviennent plutôt dans le cadre de rapports à présenter (au Parlement, à l'opinion publique) ou accompagnent l'enseignement tiré de la réalisation du projet. Ces contrôles se fondent sur les cinq grands critères suivants : efficience, efficacité, impact, pertinence et durabilité.

Il n'y a pas de recette miracle pour mesurer l'impact sur le développement. Il faut recourir à des indicateurs qui sont déterminés par les objectifs prioritaires du projet, par les résultats escomptés et par les activités menées. Ces indicateurs varient donc selon le type de projet et doivent en bonne partie être redéfinis à chaque évaluation. Des critères d'évaluation spécifiques ont par exemple été élaborés pour la SFSD et ils figurent dans une annexe au Message sur le cinquième crédit de programmes pour la coopération avec les pays du Sud. Pour évaluer l'impact des nouveaux instruments, nous utilisons notamment les indicateurs de la SFI (Banque mondiale).

Un séminaire d'une journée animé par un responsable de l'évaluation de la SFI, organisé en été de l'année dernière, a servi de point de départ pour l'élaboration des indicateurs que nous appliquons à nos nouveaux instruments. Nous préparons par ailleurs un manuel pour la mise au point d'indicateurs qui tiennent compte des diverses exigences auxquelles doivent répondre nos autres instru- 
ments. Enfin, nous voulons renoncer de plus en plus aux évaluations de projets pour estimer les programmes sectoriels et de pays, afin de mieux pouvoir mesurer l'impact de nos instruments sur le développement. Malgré ce déplacement vers «un plan d'évaluation supérieur», nous sommes néanmoins tenus (l'obligation figure notamment dans les messages fédéraux) de commencer par évaluer au moyen d'indicateurs spécifiques (pays, secteur, instrument) si les objectifs de nos instruments ont été atteints.

Quels sont les contacts entre les investisseurs, les responsables des instruments en Suisse, ainsi que les autorités et les groupements dans le pays en développement concerné?

Dans le cas des instruments de promotion du secteur privé, nos partenaires dans les pays bénéficiaires ne sont en général ni les gouvernements ni des groupements, mais plutôt des entreprises ou des gestionnaires de fonds.

\section{Quelle coordination est assurée entre ces instruments bilatéraux}

et les instruments multilatéraux de promotion des investissements (SFI et AMGI de la Banque mondiale)?

Lorsque de véritables synergies sont possibles, nous accordons une grande importance à la coordination entre les instruments bilatéraux et multilatéraux. En principe, avant de décider de lancer une nouvelle activité bilatérale, nous considérons les activités et les priorités du groupe de la Banque mondiale dans les pays concernés.

Ces derniers mois, nous sommes devenus un bailleur de fonds bilatéral de poids au sein de la SFI (nous cofinançons aujourd'hui environ 15 projets avec la SFI et avec d'autres donateurs bilatéraux). Ces activités nous ont amenés à établir des échanges réguliers avec les divers services de la SFI. Dans le courant de l'année 2000, nous avons également commencé à étendre les instruments de la promotion du secteur privé aux pays en transition, et nous avons, dans ce cadre, intensifié notre collaboration avec la BERD.

Pour ce qui est de notre travail quotidien, il est utile que le domaine de prestations Développement et Transition soit une unité petite, dotée d'une structure simple. Toutes les collaboratrices peuvent ainsi se faire une idée assez précise de l'ensemble des activités du Seco. En effet, tant dans le cadre de nos activités bilatérales qu'au sein des organes multilatéraux, nous cherchons à promouvoir la coopération et à éviter le travail à double. Les facilités régionales de la Banque mondiale et de la SFI pour la phase préparatoire des projets sont un bon exemple pour exploiter les synergies potentielles. Nous essayons toujours de les mettre à profit pour établir des relations avec les intermédiaires financiers que nous appuyons. 


\section{PROMOUVOIR LE SECTEUR PRIVÉ - DANS QUELLES CONDITIONS?}

\section{Peter Bosshard}

Les sociétés Sri Vasavi Florex et Industries Ltd. et Prime International Private Ltd. produisent en Inde des fleurs hors sol destinées à l'exportation. L'eau chargée d'engrais et de pesticides est conduite dans des bassins, d'où elle s'infiltre dans la terre afin (comme le note un bref aperçu des répercussions sur l'environnement) «de maintenir la nappe phréatique à un niveau correct». Egalement imprégnée de pesticides, la laine minérale servant de base nutritive est compostée après usage. Ces méthodes de culture ne sont guère compatibles avec les directives écologiques de la Banque mondiale, qui préconisent de renoncer autant que possible aux pesticides. On ne connaît pas l'écobilan du transport aérien des fleurs dans les pays de destination. Ces deux sociétés bénéficient d'une participation du Swiss Technology Venture Capital Fund (SwissTec), nouvel instrument de la coopération suisse en vue de promouvoir le secteur privé.

Ces entreprises, certes, créent des emplois. On peut toutefois se demander s'il appartient à la coopération de soutenir des firmes - et d'intervenir par conséquent sur les mécanismes du marché - qui ne se distinguent en aucune manière par des méthodes de production respectueuses de l'environnement ou innovantes. Cette question met en évidence les conflits d'intérêts que doit affronter la coopération suisse (et en particulier le Seco) lorsqu'il s'agit de promouvoir le secteur privé.

\section{$\square$ La nécessité de critères explicites}

La Déclaration de Berne examine depuis le milieu des années 90 les activités de promotion du secteur privé menées par le Groupe de la Banque mondiale et par la coopération suisse au développement. Elle a également suivi de façon active la préparation du $5^{\mathrm{e}}$ crédit de programme pour des mesures de politique économique et commerciale, et en particulier la création de la Swiss Development Finance Corporation (SDFC). (Voir le point de vue de la Déclaration de Berne à ce sujet sous www.evb.ch/eza.htm).

Aux termes de la loi fédérale de 1976, la coopération au développement a pour tâches de soutenir en priorité les efforts des pays, régions et groupes de population les plus défavorisés, ainsi que la sécurité alimentaire et l'équilibre écologique. Le cas échéant, cela peut se faire sous forme d'investissements dans des entreprises privées - raison pour laquelle la Déclaration de Berne ne rejette pas le principe d'une promotion du secteur privé par la coopération au développement. Mais les participations de ce genre doivent être assorties de critères explicites en ce qui concerne les produits à fabriquer, les modes ou les sites de production. L'argent de la coopération ne saurait servir à imiter simplement des processus inhérents au marché. Il est par exemple judicieux que la Société financière internationale (SFI) de la Banque mondiale participe à une usine qui produit des médicaments génériques en Tanzanie. Par contre, le Groupe de la Banque mondiale pourrait tranquillement laisser au seul secteur privé le soin de construire des équipements pour la mise en bouteilles de Coca-Cola ou des hôtels de luxe dans l'ex-Union soviétique ou dans des pays émergents. 


\section{$\square$ Bilan provisoire mitigé}

Comment se présente le bilan provisoire de la promotion du secteur privé par le Seco - notamment par la nouvelle SDFC - en termes de développement? On constate aujourd'hui que les investisseurs privés s'intéressent peu au capital social de la SDFC; même quand il s'agit de participer à des projets concrets, les PME helvétiques ne se bousculent pas au portillon de la nouvelle société. Comme la SDFC est l'instrument phare du crédit de programme et une question de prestige pour le Seco, les autorités fédérales ont cru qu'elles ne pouvaient pas se permettre d'abandonner ce projet ou de le remettre à plus tard. Au lieu de cela, le Seco s'est montré disposé à faire des concessions supplémentaires aux investisseurs privés: ceux-ci obtiennent par exemple des garanties fédérales pour la couverture des risques politiques auxquels sont exposés les projets soutenus, ce qui n'était pas prévu au départ.

Les pouvoirs publics participent au capital de la SDFC à hauteur de $49 \%$ et financent par ailleurs diverses prestations. L'administration fédérale se contente volontairement d'une participation minoritaire au conseil d'administration de cette société, et renonce même à siéger dans sa commission des investissements. Comme l'a justement souligné de façon critique le dernier rapport de l'OCDE sur la coopération au développement suisse, les autorités fédérales se sont ainsi privées d'un moyen d'imposer que les investissements de la SDFC respectent systématiquement les critères de la politique de développement. Et les directives qui régissent les activités de la SDFC sont très vagues dans les domaines écologique et social.

Les projets auxquels la SDFC a participé jusqu'à présent semblent valables à première vue, contrairement aux exploitations hors sol de SwissTec en Inde. Après quelques hésitations, le Seco a pris le parti d'une transparence complète pour ce qui est des projets qu'il soutient. On peut enfin considérer comme louable le fait que la société accepte le manque d'empressement manifesté jusqu'ici par les investisseurs privés, qu'elle ne cherche pas à précipiter la conclusion de projets. Il conviendra toutefois d'évaluer dans quelques années si le nombre - et, bien entendu, la qualité - des placements de la SDFC justifie l'effort financier consenti par les autorités fédérales.

Le $6^{\text {e }}$ crédit de programme pour des mesures de politique économique et commerciale se prépare au cours de l'année 2001. Pour que l'on puisse tirer des leçons appropriées du crédit de programme en cours, il faudrait que l'activité des nouvelles sociétés du Seco soit analysée par une instance indépendante dans les mois qui viennent - donc bien avant l'évaluation officielle prévue dans plusieurs années. 


\section{POINTS DE VUE DE LA COMMUNAUTÉ DE TRAVAIL DES CEUVRES D'ENTRAIDE CONCERNANT LES NOUVEAUX INSTRUMENTS}

NADINE KEIM

La Communauté de travail $(\mathrm{CT})$ a suivi de près l'élaboration du crédit-cadre $\mathrm{V}$ et la création des nouveaux instruments commerciaux et financiers. La CT a pris position à plusieurs occasions - notamment à l'égard de l'administration en décembre 1995, à l'égard des deux commissions parlementaires pour la politique économique extérieure en août et octobre 1996 et à l'égard de la presse en mai (communiqué) et en août 1996 (conférence). La CT y saluait le rôle clé des mesures du Seco dans le cadre de la coopération au développement, ainsi que la volonté du Seco d'innover avec de nouvelles mesures. Néanmoins, elle relevait un certain nombre de points critiques qui restent d'actualité à ce jour.

De manière générale, la croissance économique, le commerce et les investissements ne sont pas automatiquement synonymes de développement durable. Par conséquent, les instruments du Seco doivent mettre explicitement l'accent sur les besoins des pays en développement et promouvoir les conditions de vie de leurs populations. Voici, en résumé, les principaux points critiques selon la CT:

Pays destinataires: La CT trouve que les pays destinataires du Seco sont trop nombreux. Ils ne recouvrent que partiellement les pays de concentration de la DDC et donnent trop d'importance aux pays émergents. Déjà délicat avec des mesures de coopération classiques, ce problème le devient encore plus avec des mesures commerciales/financières, car ces dernières accroissent ainsi encore davantage la marginalisation des pays les moins avancés sur les marchés mondiaux.

Accords bilatéraux: La CT craint la multiplication des mesures du Seco dans le cadre d'accords de libre-échange «bilatéraux» entre la Suisse et certains de ses pays partenaires. Il y a un risque d'élaborer de plus en plus de mesures commerciales/financières pour des pays où elles ne sont pas réellement nécessaires, mais où la Suisse a un intérêt à faire un geste en échange de concessions en sa faveur (par exemple: pays méditerranéens).

Aide liée : La CT estime que près de la moitié des moyens financiers du créditcadre V sont liés à des biens et services suisses (contrairement au crédit précédent où l'aide liée ne représentait «que» $30 \%$ ). Alors qu'il a été maintes fois prouvé que l'aide liée rapporte surtout au pays donateur et coûte aux destinataires (choix des priorités, prix, importations aux dépens des inputs locaux, etc). Ainsi, les mesures commerciales/financières semblent promouvoir davantage l'économie suisse que l'économie des pays qu'elles devraient soutenir.

Garanties: La CT estime que près d'un tiers des moyens financiers du créditcadre $\mathrm{V}$ ne sont pas réellement dépensés, car ils se présentent sous la forme de garanties. La coopération au développement devrait au contraire favoriser les transferts nets de capitaux vers les pays défavorisés.

Société financière pour le développement: La CT trouve l'idée d'une telle société intéressante, mais pas sans risques. Des tiraillements délicats entre la rentabilité des projets et les critères de développement sont prévisibles. Autre tiraillement inévitable entre les affaires qui se feraient sans elle et les projets non rentables. 
Fonds d'égalisation: La CT s'oppose à cet instrument, car il permet uniquement aux industries suisses de rivaliser avec leurs concurrents étrangers. Un tel fonds (avec les moyens de la coopération) ne se justifie qu'en faveur d'exportateurs du Sud pour leur permettre d'être compétitifs sur les marchés internationaux.

Critères de sélection des projets: Les critères de développement devraient être contraignants et basés sur la Loi fédérale sur la coopération au développement du 19 mars 1976. Les principes et objectifs de celle-ci (art. 2 et 5) devraient être prépondérants dans le financement des mesures du Seco. La loi prévoit notamment l'offre de biens et services nécessaires au développement aux meilleures conditions, c'est-à-dire sans tenir compte de la source d'approvisionnement (aide déliée).

Répartition géographique des projets: Une sélection de pays défavorisés doit être clairement définie par le Seco, en étroite collaboration avec la DDC. Il n'est ni crédible ni efficace de vouloir aider un peu tous les pays. L'OCDE a critiqué de nombreuses fois la trop grande dispersion de la coopération suisse. Les marchés difficiles des pays les moins avancés, qui ont tant de peine à attirer les investissements privés, doivent également obtenir une chance. Reste que le gros des projets revient logiquement aux pays intermédiaires, pays dans lesquels les investisseurs hésitent à s'aventurer seuls.

Evaluation de l'impact sur le développement: La CT souhaite que les nouvelles mesures du Seco soient évaluées avec soin par une instance indépendante. L'impact sur le développement de ces mesures est difficile à cerner, notamment en raison du manque de recul depuis la création de ces instruments, mais aussi parce que les critères de développement sont souvent peu précis. Il s'agit ainsi d'examiner ces instruments sur la base de la Loi fédérale sur la coopération au développement, ainsi que sous l'angle de la réduction de la pauvreté. Rien ne permet actuellement de garantir que les préoccupations de développement sont prépondérantes dans le choix des projets.

Contacts entre les différents acteurs: La CT souhaiterait être davantage informée et associée aux nouvelles mesures du Seco. Elle est prête à soutenir de près la réalisation de celles-ci, à certaines conditions, et a exprimé à plusieurs reprises son intérêt à participer concrètement à la mise sur pied de la Société financière pour le développement (au niveau du conseil d'administration), par exemple.

Coordination entre les instruments bilatéraux et multilatéraux: Plusieurs expériences d'instruments financiers existent au niveau bilatéral et multilatéral. Celles-ci ont montré que la promotion du secteur privé exige des critères sociaux et écologiques afin d'orienter ces investissements dans l'intérêt des pays en développement, à l'instar des institutions de la Banque mondiale (MIGA et IFC) par exemple. La Suisse soutient ces critères au niveau multilatéral, mais n'en a pas exigé dans le cadre de la Société financière pour le développement. Autre incohérence: des critères sévères ont été élaborés dans le domaine des crédits et des dettes (ajustement structurel, réduction de la pauvreté, good governance, etc), alors que dans le cas des instruments financiers du Seco, les critères sont peu clairs. Tout porte à croire que ces mesures visent avant tout la promotion de la place économique suisse... 


\section{QUESTIONS SOUS L'ANGLE DE LA POLITIOUE DE DÉVELOPPEMENT}

\section{Gérard Perroulaz*}

- Faut-il utiliser des fonds de l'APD

pour encourager les investissements de PME suisses?

Les instruments ont leur place dans une zone qui se situe entre la promotion de l'économie privée suisse (soutien aux PME) et la promotion du secteur privé dans les pays en développement.

Les Directives pour l'établissement des statistiques du Comité d'aide au développement permettent de faire une distinction claire entre plusieurs types de flux financiers à destination des pays en développement: l'Aide publique au développement (APD), les autres apports du secteur public, les dons des organismes privés bénévoles et les apports du secteur privé (investissements directs, flux bancaires, etc.)

Rappelons à ce titre la définition donnée par le CAD pour les flux qui peuvent être compris dans l'agrégat statistique d'aide publique au développement ${ }^{14}$ (souligné par l'auteur):

«On entend par aide publique au développement» tous les apports de ressources qui sont fournis aux pays de la liste $\mathrm{I}$ du $\mathrm{CAD}$, ou à des institutions multilatérales pour être ensuite acheminés vers ces pays, et qui répondent aux critères suivants:

- Emaner d'organismes publics ou d'organismes agissant pour le compte d'organismes publics

Sachant que chaque opération doit en outre:

- Avoir pour but essentiel de favoriser le développement économique et l'amélioration du niveau de vie des pays bénéficiaires de l'aide.

- Etre assortie de conditions favorables et comporter un élément de libéralité au moins égal à $25 \%$.»

Les autres apports du secteur public comprennent par exemple les mesures suivantes (souligné par l'auteur):

- Les dons qui sont accordés à des pays bénéficiaires de l'aide à des fins de représentation ou essentiellement commerciales.

- Les opérations bilatérales du secteur public qui ont pour but essentiel de favoriser les exportations (par exemple les crédits à l'exportation).

- Les subventions (dons) accordées au secteur privé pour lui permettre d'assouplir les conditions des crédits qu'il consent aux pays bénéficiaires de l'aide.

- Les aides au financement d'investissements privés.

Ces autres apports du secteur public ne devraient donc pas être inclus dans l'APD. «Les prêts ou investissements (prises de participation) effectués dans le cadre de coentreprises avec des pays bénéficiaires par des organismes publics et ne répondant pas aux conditions requises pour être classés dans l'APD » doivent donc être inclus dans cette rubrique des autres apports du secteur public ${ }^{15}$. Sont

14. Source: OCDE, Comité d'aide au développement (CAD), Directives pour l'établissement des rapports statistiques au CAD, Paris, OCDE, 4 avril 2000.

15. Directives du CAD, p. 24. 
aussi compris dans cette rubrique les "prêts et dons consentis par le secteur public à des sociétés privées établies dans le pays donneur pour contribuer à financer un investissement déterminé dans un pays bénéficiaire de $1^{\prime}$ aide ${ }^{16}$. Les concours apportés à des programmes généraux d'investissement réalisés par des entreprises ne doivent pas être pris en compte bien que ce genre d'action puisse indirectement encourager l'investissement dans les pays en développement ${ }^{17}$.

Les investissements directs des entreprises privées sont à classer dans un autre agrégat statistique des flux financiers, les «apports du secteur privé aux conditions du marché».

Ces définitions du CAD sont assez claires dans ce domaine et permettent par exemple d'inclure la part de don fournie par la Confédération pour un financement mixte dans l'APD, mais de ne pas inclure dans l'APD la part du crédit accordée par un consortium de banques aux conditions du marché.

Les nouveaux instruments décrits ci-dessus relèvent à l'évidence d'une zone qui peut se situer entre l'APD et les autres apports du secteur public (part de soutien public associée à un flux d'investissement privé). Le seul fait souvent invoqué par le Seco que ces investissements créent des emplois, et donc favorisent la croissance économique, ne saurait en aucun cas suffire à inclure ces soutiens dans l'APD (car toute entreprise suisse crée des emplois ou sauvegarde certains emplois en investissant dans une filiale ou en rachetant des entreprises qui disparaîtraient sans ce rachat). Une distinction devrait en fait être établie selon les types d'instruments. Les instruments favorisant les transferts de technologies environnementales (du type des Cleaner Production Centers) et l'approvisionnement par le Seco de certains fonds situés dans un pays en développement relèvent sûrement de l'APD. Inclure dans l'APD des prises de capital dans des sociétés situées en Suisse et servant prioritairement à encourager les PME suisses à investir à l'étranger semble par contre plus contestable. Ces mesures, tout comme la GRE, seraient plutôt assimilables à la catégorie de flux financiers «autres apports du secteur public».

Ce débat de savoir s'il est judicieux ou non de prendre de l'argent sur des crédits de programme de coopération au développement pour appuyer des investissements n'enlève bien sûr rien à l'intérêt de ces instruments novateurs pour les PME suisses désireuses de s'implanter dans un pays en développement et de l'intérêt évident pour une entreprise du Sud de pouvoir avoir accès à certains financements pour moderniser ses installations.

\section{$\square$ Dispersion des moyens de la coopération} au développement sur un nombre d'instruments très élevé?

Concernant la Société financière suisse pour le développement (SDFC) l'examen de la politique suisse de coopération par le CAD soulevait le problème suivant: «On peut toutefois s'interroger sur le fait que le Seco soit minoritaire au capital et au Conseil de la SDFC et ne participe pas à sa gestion opérationnelle, déléguée à des consultants, alors qu'il devra prendre en charge une partie importante des déficits, inévitables au cours des premières années de fonctionnement. Le Seco et la société de gestion se sont mis d'accord sur les critères de

16. La SOFI et la SDFC sont des société ou fondation basées en Suisse.

17. Directives du CAD, p. 25. 
la politique d'investissement, mais la participation du Seco au seul conseil d'administration ne peut garantir que les préoccupations de développement seront toujours prépondérantes dans les décisions de gestion. $»^{18}$ Le CAD se pose aussi la question de savoir s'il ne serait pas préférable de transférer à la SDFC les vingt autres participations du Seco dans des fonds qui sont actuellement gérés directement, sans grands moyens, par le Seco (portefeuille de vingt intermédiaires financiers lourd à gérer en regard des ressources humaines limitées du Seco).

Laurent Guye relevait, en 1997 déjà, un risque de dispersion des efforts: «Le nouveau partage des rôles avec le secteur privé implique que l'initiative revienne de plus en plus à ce dernier (...). C'est au secteur privé de proposer les projets concrets. Voilà qui n'est pas sans conséquences sur l'organisation du travail et sur nos rapports avec les gouvernements des pays bénéficiaires. Poussé à l'extrême, cet abandon de la logique planificatrice pourrait signifier que nous nous retrouvions engagés simultanément dans un trop grand nombre de pays et avec trop de lignes d'action ou d'instruments différents, ce qui serait préjudiciable à notre capacité d'accompagnement et à l'efficacité. Un compromis doit bien sûr être trouvé entre la flexibilité totale, source de dispersion, et la culture de la planification rigide qui prévaut encore dans bien des agences d'aide. $\gg^{19}$

\section{$\square$ Dispersion des moyens dans d'autres pays} que les pays de concentration de la coopération?

On peut comprendre que les nouveaux instruments de promotion des investissements ne s'adressent pas, par leur nature même, prioritairement aux pays les plus pauvres, mais plutôt à des pays à revenu intermédiaire. Ceci explique qu'il n'y a pas forcément concordance entre la liste des pays de concentration de la coopération suisse et les pays visés par ces instruments. La description des instruments ci-dessus montre par contre que les différents instruments s'adressent à un nombre élevé de pays, différents selon les types d'instruments et qu'il y a effectivement une grande dispersion géographique.

\section{$\square$ Transparence sur les projets financés}

L'information sur les projets financés par le biais de ces instruments et sur les entreprises qui ont bénéficié de l'aide est disponible de manière encore très fragmentaire. Ceci s'explique toutefois en partie par le fait que ces instruments sont encore jeunes.

\section{$\square$ Critères de choix des projets à financer}

Les projets à financer doivent bien sûr répondre à des critères de viabilité financière, mais puisque ces instruments sont financés par des fonds de coopération au développement, quels sont les critères environnementaux et sociaux mis en avant dans le choix des projets d'investissement? c'est l'une des questions développées lors de l'atelier d'évaluation sur les instruments du Seco (voir compte rendu ci-dessus).

18. Comité d'aide au développement, examen en matière de coopération pour le développement. Suisse. Paris, OCDE, Préimpression des dossiers du CAD 2000, vol. 1, nº 4.

19. Laurent Guye, «Coopération au développement: Pour un vrai partenariat entre la Confédération et le secteur privé», in Annuaire Suisse-Tiers Monde 1998, pp 122-123. 


\subsection{AUTRES INSTRUMENTS DE PROMOTION DES INVESTISSEMENTS}

\section{Gérard Perroulaz}

Nous présentons, dans cette partie, le fonctionnement des instruments de promotion des investissements du Seco, en donnant des exemples concrets de projets soutenus grâce à ces instruments.

\section{APPUIS AUX PME}

\section{$\square$ Mekong Project Development Facility (MPDF)}

Cet instrument de soutien aux PME a été créé en 1997. Il est actif au Vietnam (son siège est à Hanoi), au Laos et au Cambodge. Son objectif est de créer des liens entre les PME et les institutions financières domestiques et étrangères, pour favoriser l'accès à du capital d'investissement ${ }^{20}$. Les activités comprennent l'assistance technique, la conduite d'études de faisabilité et d'analyses financières, la promotion de partenariats entre les entreprises locales et des partenaires financiers, techniques et commerciaux (domestiques ou étrangers). Le MPDF est géré par la Société financière internationale, filiale de la Banque mondiale et financé par plusieurs pays donateurs (dont la Suisse) et des institutions financières multilatérales.

\section{- Service de promotion}

et de développement des investissements en Afrique (APDF)

Ce service a été créé en 1986 par la Banque africaine de développement, la Société financière internationale (SFI), le PNUD et quinze pays bailleurs de fonds. L'APDF ${ }^{21}$ a pour objectif d'aider les entrepreneurs africains et de promouvoir les PME (conseils pour le démarrage de nouvelles entreprises, assistance technique et assistance en gestion). Sans financer lui-même les projets, il aide les entrepreneurs à obtenir un financement des banques ou d'autres sources.

\section{FONDS DE PARTENARIAT}

\section{$\square$ Dans le cadre du partenariat sino-suisse}

Le Sino-Swiss Partnership Fund (SSPF), créé le 11 décembre 1997, est un instrument de financement de projets d'investissements directs (sous forme de capitalrisque, de prêts ou de garanties), avec pour but d'établir des relations de partenariat entre des entreprises prioritairement suisses (mais aussi d'autres pays de l'OCDE) et des entreprises chinoises, notamment par le biais d'entreprises conjointes. Le fonds permet d'acquérir une participation minoritaire pour une période limitée (investissement maximal de 5 millions de francs pour un projet).

Il permet d'ouvrir les portes de la Chine aux PME de Suisse. C'est une initiative conjointe de la Confédération suisse et de la République populaire de Chine. La Confédération a apporté une contribution de 25 millions de francs suisses au fonds. 


\section{$\square$ Dans le cadre du partenariat tuniso-suisse}

La ligne de crédit ouverte dans le cadre du partenariat tuniso-suisse a pour but de soutenir les projets d'investissement de PME tuniso-suisses installées en Tunisie (joint-venture). Le fonds permet par exemple de procurer des crédits (de 100 '000 francs à 2 millions de francs au maximum) servant à financer:

- des importations de biens d'équipement et/ou de services d'origine suisse utilisés dans le projet d'investissement;

- des fonds de roulement liés au projet d'investissement;

- une partie des fonds propres que doivent apporter les promoteurs tunisiens à la coentreprise :

- la participation d'une banque tunisienne qui envisage de s'impliquer comme actionnaire ou copropriétaire dans la coentreprise tuniso-suisse.

Pratiquement, les demandes de financement sont transmis pour approbation au Seco (via l'ambassade de Suisse à Tunis).

\section{INSTRUMENTS FINANCIERS - FONDS DE CAPITAL-RISQUE}

L'investissement doit être viable et rapporter aux investisseurs le revenu désiré, ce qui ne dépend pas seulement des efforts de gestion et de marketing, mais aussi de la structure financière de l'entreprise (solide base de capital propre à même de garantir la croissance). Les conditions de financement pour des projets d'investissement locaux sont souvent très peu avantageuses. Une jeune entreprise est souvent confrontée au problème suivant: Comment financer une expansion de son entreprise, un nouvel équipement? Les formes traditionnelles de financement telles que les crédits bancaires sont coûteuses (intérêt élevé et risque considéré souvent comme étant trop grand pour les institutions bancaires). C'est là que des institutions fournissant du capital-risque sont très utiles. Elles permettent d'augmenter le capital de base pour l'entreprise (fonds propres, donc sans intérêt). L'institution fournissant le capital-risque va donc prendre part à la gestion de l'entreprise, de manière temporaire (vente des parts au capital à la fin du soutien par exemple).

Le Seco participe à toute une série de fonds de capital-risque pour aider l'investisseur potentiel et le partenaire dans un pays en développement ou un pays de l'Europe centrale et orientale.

\section{$\square$ Afrique du Sud: «Msele NedVentures Ltd.»}

C'est un fonds de capital-risque qui prend des participations dans des PME d'Afrique du Sud. Le fonds vise de préférence des entreprises à haut potentiel de croissance et favorise en priorité celles qui ont été lancées par la nouvelle classe commerçante issue de la communauté noire d'Afrique du Sud. Le fonds privilégie les transferts de technologies et de ce fait, il est intéressant pour les entreprises étrangères voulant lancer des projets en Afrique du Sud.

Le Seco est actionnaire de «Msele NedVentures Ltd.» au côté de la Proparco (filiale de la Caisse française de développement) et de la DEG (Deutsche Investitions- und Entwicklungsgesellschaft).

Types d'entreprises soutenues par le Msele NedVentures:

- petits cultivateurs de roses pour l'exportation; 
- manufacture d'engrais solubles dans l'eau;

- coentreprise sud-africano-suisse qui fabrique du matériel d'emballage pour l'industrie pharmaceutique;

- fabricant de glaces;

- fabricant de verre pour l'industrie automobile;

- entreprise sud-africano-française fabriquant et vendant des baguettes de pain et des viennoiseries;

- entreprise prêtant du matériel et ravitaillant des réceptions;

- entreprise développant des parcomètres électroniques;

- manufacture de produits cosmétiques.

\section{$\square$ Afrique de l'Ouest:}

Fonds de garantie des investissements privés en Afrique de l'Ouest (GARI)

L'expérience pilote en la matière a été lancée en 1995 en collaboration avec l'Agence française du développement, la Banque européenne d'investissement et la Deutsche Entwicklungsgesellschaft et des banques locales (participation du Seco de $16 \%$ au capital du GARI). Le GARI (situé à Lomé, Togo) offre des garanties sur des crédits à l'investissement à moyen et long terme. Le Seco peut faire bénéficier de son statut d'actionnaire (coût réduit de la garantie) des établissements financiers suisses désireux d'octroyer un crédit à l'investissement pour un projet situé dans l'un des pays de la CEDEAO (Communauté économique des Etats d'Afrique de 1'Ouest ${ }^{22}$ ).

\section{- Inde et pays riverains de l'océan Indien:} fonds pour la promotion technologique SwissTec VCF

Le Seco a parrainé le fonds suisse de capital-risque pour la promotion technologique SwissTec VCF. Pour le moment, l'Inde a été le seul et unique bénéficiaire. Cet instrument permet de fournir des fonds propres et fonds assimilables à des fonds propres dans des PME. Il donne à des entreprises locales (qui entretiennent des liens étroits avec les entreprises suisses) les moyens de développer des projets qui introduisent des technologies ou des processus de production inédits dans des domaines variés tels que la santé (installations et équipements médicaux, médicaments), technologies vertes (utilisation plus économe des ressources), l'industrie alimentaire, les emballages et les arts graphiques. Le Seco a fondé ce fonds dont le siège est à l'île Maurice, qui est représenté au conseil d'administration et a fourni les 18 premiers millions de dollars nécessaires. Des moyens supplémentaires seront levés auprès d'autres investisseurs institutionnels et privés suisses, avec le but d'arriver à un capital social de 60 millions de dollars.

Types de projets soutenus:

- fabricant d'emballages utilisant un équipement et une technologie issus d'une entreprise suisse;

- unité de production de fil de coton dans une entreprise indienne qui utilise de l'équipement suisse;

- fabrique de tricots (entreprise conjointe indo-suisse et Italie);

22. CEDEAO: Bénin, Burkina Faso, Cap-Vert, Côte d'Ivoire, Gambie, Ghana, Guinée, Guinée-Bissau, Liberia, Mali, Mauritanie, Niger, Nigeria, Sénégal, Sierra Leone, Togo. 
- floriculture high-tech: production hors sol, dans des serres contrôlées par les technologies de l'information, axée sur l'exportation à destination de distributeurs suisses de chaînes de supermarchés;

- production de sel de table iodé (entreprise conjointe indo-suisse)

\section{$\square$ Inde: Infrastructure Development Finance Corporation (IDFC)}

Cette institution financière spécialisée dans le financement des infrastructures a été créée au début de l'année 1998 avec un capital initial de 250 millions de dollars environ, dont $40 \%$ détenus par des investisseurs internationaux (10 millions de dollars du Seco). Elle participe au financement de projets d'infrastructure (prises de participation en capital, octrois de financements à long terme, garanties), notamment dans les secteurs de l'énergie et des télécommunications.

\section{$\square$ Europe de l'Est: fonds pour l'environnement}

Fonds de capital-risque pour les investissements dans le domaine de l'environnement en Europe de l'Est (CEEEIF), fondé en décembre 1997 par la Banque européenne pour la reconstruction et le développement (BERD), avec la participation de la Suisse. Le CEEEIF investit dans des entreprises qui fabriquent des biens et des équipements importants sur le plan environnemental, qui fournissent des services dans ce domaine ou qui possèdent des installations (stations d'épuration et décharges), dans des entreprises privées ou des entreprises d'Etat dont la privatisation est imminente. Les pays d'intervention prioritaires sont la Hongrie, la Pologne, la République tchèque, la Slovaquie et la Roumanie. Le fonds a démarré avec un capital de 22 millions d'euros, dont 2 du Seco, mais l'objectif est d'intéresser d'autres investisseurs pour atteindre un capital de 50 millions d'euros.

\section{Amérique latine: fonds pour la biodiversité. Terra Capital Investors Ltd.}

Créé en 1998 à Sao Paulo (siège juridique dans les îles Vierges britanniques), c'est le premier fonds mondial de capital-risque pour les investissements dans la sauvegarde de la biodiversité. Il a été créé à l'initiative de la Société financière internationale (SFI) du groupe de la Banque mondiale (capital de 15 millions de dollars, dont 3 millions financés par la Suisse). Un représentant du Seco siège au conseil d'administration et un représentant de l'OFEFP au comité de la biodiversité. Le Seco a aussi délégué le président de la Chambre de commerce Suisse-Brésil au comité des investissements. Le fonds permet de renforcer le capital propre de PME d'Amérique latine et de drainer des capitaux privés pour investir dans des domaines ayant trait à la diversité biologique.

Un fonds complémentaire de 5 millions de dollars (alimenté par le FEM) permet le financement de mesures d'accompagnement non rentables pour réduire les coûts de transaction élevés liés aux investissements dans la biodiversité (études de faisabilité, évaluations techniques et de risques, études d'impact des activités des entreprises sur la biodiversité).

Le fonds est essentiellement destiné à renforcer le capital propre de petites et moyennes entreprises d'Amérique latine qui ne sont pas cotées en bourse, et accessoirement à drainer des capitaux privés pour investir dans des domaines ayant trait à la diversité biologique. 
$\square$ Amérique latine: ProFunds International SA

C'est une société d'investissement qui engage des capitaux dans des sociétés de crédit aux petites entreprises en Amérique latine. Elle a été fondée en 1995 au Costa Rica (enregistrée à Panama) et est dotée d'un capital social de 22 millions de dollars (avec une participation du Seco, membre fondateur et principal actionnaire, à hauteur de 3,2 millions de dollars). Les actionnaires sont des institutions bilatérales ou multilatérales, mais aussi des ONG telles que la Fondation suisse FUNDES.

Exemple de soutien: Banco Solidario SA (Bancosol) en Bolivie, institution financière qui accorde des crédits à des PME et qui a pris une très grande importance (un crédit sur trois octroyés en Bolivie est actuellement le fait de Bancosol).

\section{$\square$ Corporation Financiera Ambiental}

Fonds pour la protection de l'environnement en Amérique centrale.

Créé en 1996 (siège au Costa Rica), c'est le premier fonds mondial de capitalrisque pour le financement des investissements privés dans la protection de l'environnement (capital de 12 millions de dollars, avec une participation de la Suisse à hauteur de 2,5 millions de dollars). La Suisse est représentée dans le conseil d'administration et dans le comité des investissements. Il finance, par le biais de participation en actions ou crédits, la rénovation ou l'extension de PME.

Exemple de soutien: une entreprise hondurienne tournée vers l'exportation spécialisée dans le recyclage de l'aluminium (aux USA). Le crédit a permis d'agrandir les installations de production.

\section{SOURCE}

Site Internet du Seco: www.seco-admin.ch. 